\title{
on A global DifFerential geOMETRIC APPROACH TO THE RATIONAL MECHANICS OF DEFORMABLE MEDIA
}

\author{
E. Binz - D. Socolescu
}

Nr. $84 / 1988$

\section{Table of Contents}

1. The space of configurations as a principal bundle

2. The metric $\mathfrak{B}_{E}$ on the configuration space $E\left(M, \mathbb{R}^{n}\right)$

3. The constitutive law and the general equations of motion

4. The motion along a fixed surface $i(M) \subset \mathbb{R}^{n}$

5. The $\mathbb{R}^{\mathrm{n}}$-invariance of internal constitutive laws

6. On the characterization of $\mathbb{R}^{\mathrm{n}}$-valued one-forms relative to embeddings

7. The equations of motion of a deformable medium subjected to an internal constitutive law

8. The structural viscosity

9. The equations of motion of a deformable medium subjected to a general constitutive law 


\section{Introduction}

In the past the rational mechanics of deformable media was largely concerned with materials governed by linear constitutive equations. In recent years, the theory has expanded considerably towards covering materials for which the constitutive equations are inherently nonlinear, and/or whose mechanical properties resemble in some respects those of a fluid and in others those of a solid (cf[ $\mathrm{Tr}, \mathrm{No}]$,[Le,Fi]).

In the present article we formulate a satisfactory global mathematical theory of moving deformable media, which includes all these aspects.

As we shall see, in our theory the stress tensor is neither necessarily local nor symmetric. In fact it does not even determine the equations of motion. It is a more general object, namely, the stress form, which governs the motion. Typical for our considerations is the study of the motion of a soap bubble, i.e. of a closed, deformable, two-dimensional material surface in $\mathbb{R}^{3}$. It is intuitively clear that this complex motion can be described as the superposition of two different ones. These are on one hand the "elastic" deformation of the soap bubble in "radial" direction, and the "instantaneous", "viscous" fluid flow of the same soap bubble along its surface, that is "transversally" to its "elastic" deformation on the other.

For our general case let us assume that at any instant the deformable medium in $\mathbb{R}^{\mathrm{n}}$ forms a manifold and that the diffeomorphism type of this manifold does not change. Hence these manifolds are all diffeomorphic to a fixed one, which we denote by $M$.

As we shall show, this fascinating representative problem of mechanics of continua as well as the general problem of motion of a deformable medium leads to a dynamical system on a suitably chosen infinite-dimensional manifold. In order to explain the main ideas of our global approach we introduce at first the differential geometric framework.

The manifold $M$ is supposed to be smooth, compact, oriented and of dimension less or equai to $n-1$. The ambient euclidean space $\mathbb{R}^{n}$ is assumed to be equipped with a fixed scalar product $\langle$,$\rangle .$

Hence an instantaneous configuration of the medium is given by a smooth embedding of $M$ into $\mathbb{R}^{n}$. Therefore the configuration space is $E\left(M, \mathbb{R}^{n}\right)$, the space of all smooth embeddings of $M$ into $\mathbb{R}^{n}$. As shown in $[B i, F i], E\left(M, \mathbb{R}^{n}\right)$ can be given a smooth principal bundle structure. Nore precisely let Diff $\mathbb{M}$ be the group of smooth diffeomorphisms of $\mathrm{H}$, and define the action $\Phi$ of Diff $M$ on $E\left(M, \mathbb{R}^{n}\right)$ as follows

$$
\Phi(j, g)=j \circ g, \quad \forall j \in E\left(M, \mathbb{R}^{n}\right), g \in \operatorname{Diff} M .
$$

Let us denote the quotient of $E\left(M, \mathbb{R}^{n}\right)$ by this action by $U\left(H, \mathbb{R}^{n}\right)$, and identify it with the set of all smooth submanifolds of $M$ in $\mathbb{R}^{n}$ diffeomorphic with $M$. Further denote by $\Pi$ the projection of $E\left(M, \mathbb{R}^{n}\right)$ onto $U\left(M, \mathbb{R}^{\mathrm{r}}\right)$. Endowed with the $C^{\infty}$-topology, $E\left(M, \mathbb{R}^{n}\right), U\left(M, \mathbb{R}^{n}\right)$ and Diff $M$ become Frechet manifolds. The quadruple $\left(E\left(M, \mathbb{R}^{n}\right), \Pi, U\left(M, \mathbb{R}^{n}\right)\right.$, Diff $\left.M\right)$ is then a principal bundle with Diff $M$ 
as its structure group. Hence the fibres of this principal bundle have the form

$$
j \circ \operatorname{Diff} M, j \in E\left(M, \mathbb{R}^{n}\right) \text {. }
$$

In the particular case of the soap bubble we now visualize the two motions described above as follows :

The "instantaneous" fluid flow along its surface is described by a curve in one of the fibres of the above principal bundle, while the "radial" deformation is given by a curve which is transverse to the fibres of $E\left(M, \mathbb{R}^{n}\right)$.

Each configuration $j \in E\left(M, \mathbb{R}^{n}\right)$ yields a Riemannian metric $m(j)$, assuming on any pair of tangent vectors $v, w \in T M$ the value

$$
m(j)(v, w):=\langle d j \ddot{v}, d j w\rangle,
$$

where the scalar product is to be taken pointwise.

The "instantaneous" metrical properties of the moving body are described in this metric. Suppose now that the deformable medium is moving. We furnish the description of its motion by assuming that we know the work done by the forces acting upon $M$. It is in this work that all the constitutive information on the medium is coded. We therefore call it the constitutive law. The fluid component of the medium is expressed through the dependence of the work on an extra parameter. Accordingly the constitutive law is then given by

$$
F: C^{\infty}\left(M, \mathbb{R}^{n}\right) \times E\left(M, \mathbb{R}^{n}\right) \times C^{\infty}\left(M, \mathbb{R}^{n}\right) \longrightarrow \mathbb{R},
$$

where $F$ is linear in the third argument, the first factor in the cartesian product is the space of extra parameters and furthermore the trivial tangent bundle $T E\left(M, R^{n}\right)=E\left(M, R^{n}\right) \times C^{\infty}\left(M, \mathbb{R}^{n}\right)$ is the phase space of motions in $E\left(\mathbb{U}, \mathbb{R}^{\mathrm{n}}\right)$.

We concentrate on those constitutive laws which admit an integral representation. More precisely, we assume that $F$ is given by

$$
F(k)(j, h)={ }_{M} \int\left\langle\varphi_{F}(j, k), h\right\rangle \mu(j), \quad \forall j \in E\left(M, \mathbb{R}^{n}\right), h, k \in C^{\infty}\left(M, \mathbb{R}^{n}\right),
$$

with $\varphi_{F}: T E\left(M, \mathbb{R}^{n}\right) \longrightarrow C^{\infty}\left(M, \mathbb{R}^{n}\right)$ being a smooth map called the force density. The equation of motion on $E\left(M, \mathbb{R}^{n}\right)$ described by a smooth curre

$$
\sigma:(-\lambda, \lambda) \longrightarrow E\left(M, R^{n}\right), \quad \lambda>0
$$

is given then by

$$
\begin{aligned}
F(\dot{\sigma}(t))(\sigma(t), h) & ={ }_{M} \int\left\langle\varphi_{f}(\sigma(t), \dot{\sigma}(t)), h\right\rangle \mu(\sigma(t)) \\
& ={ }_{M} \int \rho(\sigma(t))\langle\dot{\sigma}(t), h\rangle \mu(\sigma(t)) \quad \forall h \in C^{\infty}\left(\mathbb{M}, \mathbb{R}^{n}\right) .
\end{aligned}
$$


We note that in $(0.7)$ we have assumed for simplicity that the constitutive law $F$ depends on the "velocity" $\dot{\sigma}(t)$, i.e. $k=\dot{\sigma}(t)$. Interpreting $h$ as a virtual displacement, $(0 . \bar{i})$ is just d'Alembert's principle of virtual work, which was formulated for the mechanics of continua by [He]. But (0.7) implies easily

$$
\rho(\sigma(t)) \ddot{\sigma}(t)=\varphi_{F}(\sigma(t), \dot{\sigma}(t)), \quad \forall t \in(-\lambda, \lambda) .
$$

To obtain a more refined form, let us denote by "I" and " $\perp$ " respectively the tangential and the normal component with respect to $\sigma(t)(\mathrm{M})$. The equation of motion (0.8) splits into the coupled system

$$
\left\{\begin{array}{l}
\nabla(\sigma(t))_{Z(t)} Z(t)+Z(t)+W\left(\sigma(t), \dot{\sigma}(t)^{\perp}\right) Z(t)+\left[\left(\dot{\sigma}(t)^{\perp}\right)^{\cdot}\right]^{T} \\
=\rho^{-1}(\sigma(t)) Y(\sigma(t), \dot{\sigma}(t)), \\
\left.\left[(\dot{\sigma}(t))^{\perp}\right)\right]^{\perp}= \\
\rho^{-1}(\sigma(t)) \varphi_{F}(\sigma(t), \dot{\sigma}(t))^{\perp}-\left[d \dot{\sigma}(t)^{\perp} Z(t)\right]^{\perp}-S(\sigma(t))(Z(t), Z(t)) .
\end{array}\right.
$$

Here $\nabla(\sigma(t))$ denotes the Levi-Civita connection of $m(\sigma(t))$, the metric given by $\sigma(t), S(\sigma(t))$ is the second fundamental tensor, $Z(t)$ and $Y(\sigma(t), \dot{\sigma}(t))$ belong to $\Gamma$ TM. Furthermore $W(j, i)$ is the unique bundle map of TM associated with a smooth map $N: M \longrightarrow \mathbb{R}^{\mathrm{n}}$ satisfying

$$
\langle\operatorname{dj} X(p), N(p)\rangle=0
$$

$\forall X \in \Gamma T M, p \in M$

and which is determined by

$$
\operatorname{dj} W(j, N) Y=(d N, Y)^{T} \text {. }
$$

Among the force densities acting on $\mathrm{M}$ we distinguish between internal forces and external ones. Of a special interest is the study of the motion of the deformable medium $M$ subjected to an internal force density. Clearly, internal physical properties of the moving medium are described by constitutive laws invariant, under the translation group $\mathbb{R}^{n}$. Evidently, the $\mathbb{R}^{n}$-imvariant configurations are differentiais of embeddings. We hence identify

$$
E\left(M, \mathbb{R}^{n}\right) / \mathbb{R}^{n} \text { with }\left\{d j \mid j \in E\left(M, \mathbb{R}^{n}\right)\right\}
$$

and more generally

$$
C^{\infty}\left(M, \mathbb{R}^{n}\right) / \mathbb{R}^{n} \text { and } \text { dh } \mid h \in C^{\infty}\left(M, \mathbb{R}^{n}\right) ?
$$

The phase space for the $\mathbb{R}^{n}$-invariant motion is hence

$$
T\left(E\left(M, \mathbb{R}^{n}\right) / \mathbb{R}^{n}\right)=\left\{d j \mid j \in E\left(M, \mathbb{R}^{n}\right)\right\} \times\left\{d h \mid h \in C^{\infty}\left(M, \mathbb{R}^{n}\right)\right\}
$$

We require that the internal constitutive law $F$ admits the representation 
where

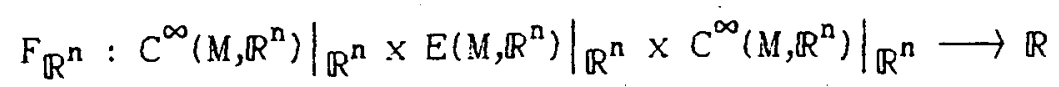

is a parameter depending one form ( the parameter varies in the front factor in (0.16)) and $T d$ is the tangent map of the differential

$$
d: E\left(M, \mathbb{R}^{n}\right) \longrightarrow E\left(M, \mathbb{R}^{n}\right) / \mathbb{R}^{n}
$$

To get a detailed description of the motion of the deformable medium, we assume now that $F_{\mathbb{R}^{n}}$ itself has an integral representation

$$
\begin{aligned}
& F_{\mathbb{R}^{n}}(d k)(d j, d l)={ }_{M} \int \alpha(d j, d k) d l \mu(j), \\
& \forall j \in E\left(M, \mathbb{R}^{n}\right), k, l \in C^{\infty}\left(M, \mathbb{R}^{n}\right),
\end{aligned}
$$

where $\alpha$ is an $\mathbb{R}^{n}$-valued one-form, the so-called stress form, depending itself on an extra parameter, i.e.

$$
\alpha:\left.E\left(M, \mathbb{R}^{n}\right)\right|_{\mathbb{R}^{n}} \longrightarrow A^{1}\left(M, \mathbb{R}^{n}\right)
$$

The stress form $\alpha$ decomposes naturally at each

into

$$
\left.(\mathrm{d} k, \mathrm{~d} j) \in T E\left(M, \mathbb{R}^{n}\right)\right|_{\mathbb{R}^{n}}=\left.\left.C^{\infty}\left(M, \mathbb{R}^{n}\right)\right|_{\mathbb{R}^{n} \times E\left(M, \mathbb{R}^{n}\right)}\right|_{\mathbb{R}^{n}}
$$

$$
\alpha(d j, d k)=c_{\alpha}(d j, d k) d j+d j C_{\alpha}(d j, d k)+d j B_{\alpha}(d j, d k),
$$

with $C_{\alpha}: T M \longrightarrow T M$ and $B_{\alpha}: T M \longrightarrow T M$ being smooth, strong bundle endomorphisms, which are respectively skew - and selfadjoint with respect to $m(i)$ and $c_{\alpha} \in C^{\infty}(M, s o(n))$. Here so(n) denotes the Lie algebra of the group of all proper rotations $S O(n)$. In case that $M$ is of codimension 1 , the equations of motion (0.9) read as

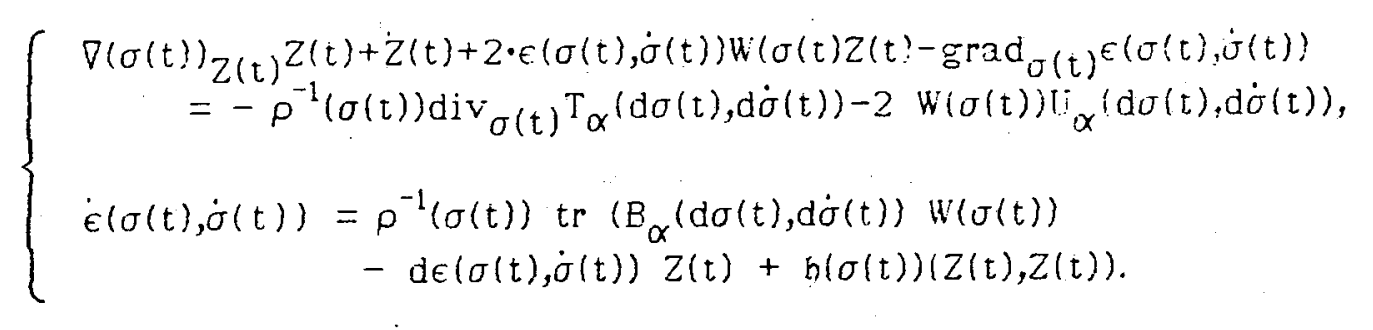

Here $\epsilon(\sigma(t), \dot{\sigma}(t)) \in C^{\infty}(M, \mathbb{R}), U_{\alpha}(d \sigma(t)) \in \Gamma T M, W(\sigma(t))$ is the Weingarten map, $\operatorname{div}_{\sigma(t)}$ is the divergence taken with respect to $m(\sigma(t)), h(\sigma(t))$ is the second fundamental form, tr denotes the trace and $T_{\alpha}(\mathrm{dj}, \mathrm{dk})$ is the so-called stress tensor, defined as

$$
T_{\alpha}(d j, d k)(X, Y)=m(j)\left(\left(B_{\alpha}+C_{\alpha}\right)(d j, d k) X, Y\right), \quad \forall Y, Y \in \Gamma T H .
$$

Each $\propto \in A^{1}\left(M, \mathbb{R}^{n}\right)$, and hence the parameter depending stress form splits 
relative to an embedding $i \in E\left(M, \mathbb{R}^{\mathrm{n}}\right)$ into

$$
\alpha=\mathrm{dh}+\beta,
$$

where $h \in C^{\infty}\left(M, \mathbb{R}^{n}\right)$, the so called integrable part of $\alpha$, is uniquely determined up to a constant. Moreover $h$ splits into parts tangential and normal to $j(M)$, i.e.

$$
\mathrm{h}=\mathrm{di} x_{\mathrm{h}}+\mathrm{h}^{\perp}
$$

(with $h^{\perp}=\Theta_{h} \cdot N(i), \Theta_{h} \in C^{\infty}\left(M, R^{n}\right)$, in case of $\operatorname{dim} M=n-1$ ) for a well determined vector field $Z_{\mathrm{h}} \in \Gamma$ TM. Using the Hodge decomposition

$$
\left\{\begin{array}{l}
X_{\mathrm{h}}=Z_{\mathrm{h}}^{0}+\operatorname{grad}_{\mathrm{i}} \psi_{\mathrm{h}} \\
\operatorname{div}_{\mathrm{i}} X_{\mathrm{h}}^{0}=0
\end{array}\right.
$$

we thus obtain immediately

$$
\begin{array}{r}
\alpha(X)=\operatorname{di} \nabla(i)_{X} X_{h}+d i W_{h}(i) X+s(i)\left(X_{h}, X\right)+\left(\mathrm{dh}^{\perp}(Y)\right)^{\perp}+\beta(X), \\
\forall X \in \Gamma T M .
\end{array}
$$

This allows us to read of the coefficients in $(0.22)$ as

$(0.27): \quad\left\{\begin{array}{l}c_{\alpha} d i=\left(d^{\perp}\right)^{\perp}+S(j)\left(X_{h}, \cdot\right)+c_{\beta} d i, \\ c_{\alpha}=\frac{1}{2}\left[\nabla(i) X_{h}^{0}-\tilde{\nabla}(i) X_{h}^{0}\right]+C_{\beta}, \\ B_{\alpha}=\frac{1}{2} L_{X_{h}^{0}}+\operatorname{grad}_{j} \psi_{h}+w_{h}(i)+B_{\beta} .\end{array}\right.$

$W_{h}(i)$ denotes here the strong smooth bundle map of TII given by

$$
\operatorname{di}_{h}(i) Z:=\left(\operatorname{dh}^{\perp}(X)\right)^{\mathrm{T}}, \quad \forall Z \in \Gamma T W
$$

$\tilde{\nabla}(i) Y_{h}$ is the adjoint of $\nabla(i) Y_{h}$ with respect to $m(i)$ formed fibrewise, so that each $v_{p} \in T_{p}$ W is sent into $\tilde{\nabla}(i) X_{h}\left(v_{p}\right), \forall p \in$ W. Woreover

$$
\mathrm{L}_{\mathrm{X}_{\mathrm{h}}}: \mathrm{TM} \longrightarrow \mathrm{TM}
$$

is the strong smooth bundle endomorphism of TM defined by the Lie derivative $L_{K_{h}}(m(i))$ via the equation

$$
m(i)\left(L_{X_{h}}, Y\right):=L_{X_{h}}(m(i))(X, Y), \quad \forall Z_{h}, X, Y \in \Gamma T M .
$$

Using now the definition of the Laplace-Beltrami operator $\Delta$ (i)

$$
\operatorname{div}_{\mathrm{i}}\left(\nabla(\mathrm{i}) X_{\mathrm{h}}\right)=\Delta(\mathrm{i}) Y_{\mathrm{h}}=-\operatorname{tr} \nabla^{2}(\mathrm{i}) Y_{\mathrm{h}},
$$

and introducing $\mathrm{R}(\mathrm{i}) \mathrm{X}_{\mathrm{h}}$ via 
where Ric(m(i)) denotes the Ricci tensor of $\mathrm{m}(\mathrm{i})$, we obtain in the case of codimension 1 the formulas

$$
\left\{\begin{aligned}
& \operatorname{div}_{i} B_{d h}= \frac{1}{2} \Delta(i) X_{h}+\frac{1}{2} R(i) Y_{h} \\
&+W(i) \operatorname{grad}_{i} \Theta_{h}+\Theta_{h} \operatorname{grad}_{i} H(i), \\
& \operatorname{div}_{i} C_{d h}= \frac{1}{2} \Delta(i) X_{h}-\frac{1}{2} R(i) X_{h}-\frac{1}{2} \operatorname{grad}_{j} \operatorname{div}_{i} X_{h}, \\
& \operatorname{tr} B_{d h}=-\Delta(i) \psi_{h}+\operatorname{tr}\left(\Theta_{h} W(i)\right)
\end{aligned}\right.
$$

a fixed embedding $i$.

Here the unnormalized mean curvature $H(i)$ is defined to be $\operatorname{tr} W(i)$. Next we introduce the notion of structural viscosity. To this end we consider on the one hand the decompositions (0.23), (0.24) and (0.25) for the stress form $\alpha(d k, d j)$, which now depends on an additional parameter dk with $k \in$ $C^{\infty}\left(M, \mathbb{R}^{n}\right)$. On the other hand, we use the decomposition for $k$, i.e.

$$
\begin{aligned}
& \mathrm{k}=\operatorname{dj} X_{\mathrm{k}}+\mathrm{k}^{\perp}, \\
& \left\{\begin{array}{l}
X_{\mathrm{k}}=X_{\mathrm{k}}^{0}+\operatorname{grad}_{\mathrm{j}} \Psi_{\mathrm{k}}, \\
\operatorname{div}{ }_{\mathrm{j}} X_{\mathrm{k}}^{0}=0 .
\end{array}\right.
\end{aligned}
$$

Even though $\mathrm{dk}$ is determined only up to a constant, $x_{\mathrm{k}}^{0}$ depends uniquely on $\mathrm{dk}$. This allows us to relate $X_{\mathrm{k}}^{0}$ and $\chi_{\mathrm{h}}^{0}$ uniquely to each other by

$$
X_{h}^{0}(d j, d k)=v(d j, d k) X_{k}^{0}+\hat{Y}_{h}(d j, d k)
$$

where $v(d j, d k) \in C^{\infty}(M, \mathbb{R})$ and $\hat{X}(d j, d k) \in \Gamma T M$ is pointwise orthogonal to $Z_{\mathrm{k}}^{0}$. We call the function $v(\mathrm{dj}, \mathrm{dk})$, the coefficient of structural viscosity.

Accordingly we call these deformable media, whose constitutive laws depend only on $k^{\perp}$, frictionless deformable media, while the deformable media, whose constitutive laws depend on the whole of $h$, will be called frictional ones.

Furnished with the structure dereloped so far, we deduce next the equations of motion of a deformable medium $\mathrm{M}$ subjected to a general constitutive law:

$$
F: E\left(M, \mathbb{R}^{n}\right) \times C^{\infty}\left(M, \mathbb{R}^{n}\right) \times C^{\infty}\left(M, \mathbb{R}^{n}\right) \longrightarrow \mathbb{R}
$$

To do this, we assume that $F$ splits into

$$
F=F_{\text {ext }}+F_{\text {int }}
$$

and that $F_{\text {int }}$ is of the form

$$
F_{\text {int }}=d^{*} F_{\mathbb{R}^{n}} .
$$


Furthermore, we require that $F_{\text {ext }}$ and $F_{\mathbb{R}^{n}}$ both admit integral representation and denote the rosulting force densities oy $\varphi_{\text {ext }}$ and $\varphi_{\text {int }}$ respectively. Using Hodge's decomposition, we obtain for all $j \in E\left(M, \mathbb{R}^{n}\right)$ and all $k \in C^{\infty}\left(M, \mathbb{R}^{n}\right)$

$$
\left\{\begin{array}{l}
\varphi_{\text {int }}(j, k)=d j \operatorname{grad}_{j} \tau_{i n t}(j, k)+d j Y_{i n t}^{0}(j, k)+\varphi_{i n t}^{\perp}(j, k), \\
\varphi_{e x t}(j, k)=d j \operatorname{grad}_{j} \tau_{e x t}(j, k)+d j Y_{e x t}^{0}(j, k)+\varphi_{e x t}^{\perp}(j, k),
\end{array}\right.
$$

and in turn

$$
\begin{aligned}
& P(j, k)=d j\left(\operatorname{grad}_{j} \tau_{i n t}(j, k)+\operatorname{grad}_{j} \tau_{\text {ext }}(j, k)\right) \\
& +d j\left(Y_{i n t}^{0}(j, k)+Y_{e x t}^{0}(j, k)\right)+P_{i n t}^{\perp}(j, k)+p_{\text {ext }}^{\perp}(j, k) \\
& :=d j \operatorname{grad}_{j} \tau(j, k)+d j Y^{0}(j, k)+P^{\perp}(j, k) \text {, } \\
& \forall j \in E\left(M, \mathbb{R}^{n}\right), k \in C^{\infty}\left(M, \mathbb{R}^{n}\right) .
\end{aligned}
$$

Hence the equations of motion in case of $\operatorname{dim} M=n-1$ are

$$
\begin{aligned}
& \nabla(\sigma(t))_{Z(t)} Z(t)+\dot{Z}(t)+Z \cdot \epsilon(\sigma(t), \dot{\sigma}(t)) W(\sigma(t)) Z(t) \\
& -\operatorname{grad}_{\sigma(t)} \epsilon(\sigma(t), \dot{\sigma}(t)) \\
& =\rho^{-1}(\sigma(\mathrm{t}))\left(\operatorname{grad}_{\sigma(\mathrm{t})} \tau(\sigma(\mathrm{t}), \dot{\sigma}(\mathrm{t}))-\Delta(\sigma(\mathrm{t}))\left[u(\mathrm{~d} \sigma(\mathrm{t}), \mathrm{d} \dot{\sigma}(\mathrm{t})) Z^{0}(\mathrm{t})\right.\right. \\
& \left.+\hat{Z}_{h}(\mathrm{~d} \sigma(t), \mathrm{d} \dot{\sigma}(t))+\operatorname{grad}_{\sigma(t)}{ }^{\mathrm{U}}(\sigma(t), \dot{\sigma}(t))\right] \\
& \text { - W( }(\sigma(t))\left[\operatorname{grad}_{\sigma(t)} \Theta_{h}(d \sigma(t), d \dot{\sigma}(t))+2\left(W(\sigma(t)) Z_{h}-\operatorname{grad} \Theta_{h}\right)\right] \\
& \left.-\Theta_{h}(d \sigma(t), d \dot{\sigma}(t)) \operatorname{grad} \sigma(t) H(\sigma(t)]\right) \text {, } \\
& \dot{\epsilon}(\sigma(t), \dot{\sigma}(t))=\rho^{-1}(\sigma(t))\left[-\tau_{\text {int }}(d \sigma(t), d \dot{\sigma}(t)) H(\sigma(t))\right. \\
& \text { - } \mathrm{dH}(\sigma(\mathrm{t}))\left[v(\mathrm{~d} \sigma(\mathrm{t}), \mathrm{d} \dot{\sigma}(\mathrm{t})) Z^{0}(\mathrm{t})+\hat{\mathrm{Z}}_{h}(\mathrm{~d} \sigma(\mathrm{t}), \dot{\sigma}(\mathrm{t}))\right] \\
& +\operatorname{div}_{\sigma(t)} v(\mathrm{~d} \sigma(\mathrm{t}), \mathrm{d} \dot{\sigma}(\mathrm{t})) W(\sigma(\mathrm{t})) Z^{0}(\mathrm{t}) \\
& +\operatorname{div}_{\sigma(t)} W(\sigma(t)) \hat{X}_{h}(d \sigma(t), d \dot{\sigma}(t)) \\
& \left.-\Theta_{h}(d \sigma(t), d \dot{\sigma}(t)) \operatorname{tr} W(\sigma(t))^{2}\right]+h(\sigma(t))(Z(t), Z(t)) \\
& \text { - d } \left.\epsilon(\sigma(t), \dot{\sigma}(t)) Z(t)+x_{\text {ext }}(\sigma(t), \dot{\sigma}(t))\right]
\end{aligned}
$$

where $\varphi_{\text {ext }}{ }^{\perp}(\sigma(t), \dot{\sigma}(t))=\kappa_{\text {ext }}(\sigma(t), \dot{\sigma}(t)) N(\sigma(t))$.

In case the motion follows a fixed surface $i(W) \subset \mathbb{R}^{n}$ given by a fixed embedding i $\in E\left(M, \mathbb{R}^{n}\right)$, the equation $(0.41)$ reduces to

$(0.42)$

$$
\begin{aligned}
& \nabla(\mathrm{i})_{X(\mathrm{t})} X(\mathrm{t})+Z(\mathrm{t})=\rho^{-1}(X(\mathrm{t}))\left[-\operatorname{grad}_{\mathrm{i}} \tau_{\text {int }}(Z(\mathrm{t}), Z(\mathrm{t}))\right. \\
& -\Delta(\mathrm{i})\left[v(X(\mathrm{t}), X(\mathrm{t})) X^{0}(\mathrm{t})+\hat{\dot{X}}_{\mathrm{h}}(X(\mathrm{t}), \bar{X}(\mathrm{t}))\right. \\
& \left.+\operatorname{grad}_{i} \psi(X(t), X(t))\right]-W(i)\left[\operatorname{grad}_{i} \Theta_{h}(Z(t), X(t))\right. \\
& \left.+2\left(W(\sigma(t)) X_{h}-\operatorname{grad} \Theta_{h}\right)\right]-\Theta_{h}(X(t), \not(t)) \operatorname{grad}_{i} H(i), \\
& 0=p^{-1}(X(t)) \cdot\left(-\tau_{\text {int }}(X(t), X(t)) \cdot \mathrm{H}(\mathrm{i})-\mathrm{dH}(\mathrm{i})\left[v(X(\mathrm{t}), \dot{X}(\mathrm{t})) Y^{0}(\mathrm{t})\right.\right. \\
& +\operatorname{div}_{i} v(X(t), X(t)) W(i) X^{0}(t)+\operatorname{div}_{j} W(i) \hat{X}_{h}(X(t), X(t)) \\
& \left.\left.+\hat{X}_{h}(X(t), X(t))\right]-\Theta_{h}(X(t), X(t)) \operatorname{tr} W(i)^{2}\right) \\
& +\mathfrak{h}(\mathrm{i})(X(\mathrm{t}), X(\mathrm{t}))+\mathrm{k}_{\text {ext }}\left(X(\mathrm{t}), X_{(\mathrm{X}}(\mathrm{t})\right) \text {, }
\end{aligned}
$$


where $X(t)$ is the push-forward of $Z(t)$ by $g(t) \in$ Diff $M$, i.e.

$$
X(t)=\operatorname{Tg}(t) Z(t) g(t)^{-1}, \quad \forall t \in(-\lambda, \lambda) .
$$

At the end of the paper we remark how to introduce a volume active pressure $\pi(d j, d k)$, which allows us to decompose $F(d j, d k)$ into

$$
F(d j, d k)=\bar{F}(d j, d k)-\pi(d j, d k) \cdot D V(j),
$$

where $V(j)$ denotes the volume of $j(M)$.

$\pi(d j, d k) \cdot D V(j)$ is the work used against the infinitesimal volume change by $D V(j)$. Let us point out that $\pi(d j, d k)$ is not identical with $\tau_{\text {int }}(d j, d k)$, the former is a real, the latter a smooth function.

We have omitted to discuss the influence of thermodynamics to the deformations of the medium. We will do these studies in a forthcoming paper.

\section{Acknowledgement}

We are indebted to M. Epstein, who has told us how to use d'Alembert's principle as a constitutive law years ago. We are especially thankfull to J. Sniatycki, who showed us the present formulation of d'Alembert's principle used in our treatment and who discussed with us some fundamental aspects of the Navier-Stokes equation.

The first author thanks the Lniversity of Calgary for its hind hospitality during his stay in fall 1987 when parts of these notes were written. 
As already mentioned a configuration of the moving deformable medium $M$ is described by a smooth embedding of $M$ into an euclidean ambient space $\mathbb{R}^{n}$. In the present paper we assume that the dimension $\operatorname{dim} M$ of the manifold $M$ satisfies the inequality

$$
\operatorname{dim} M \leq n-1 .
$$

Let us recall that a smooth embedding $j: M \longrightarrow \mathbb{R}^{n}$ is a smooth map satisfying the following conditions (i) the tangent map

$$
T j(p): T_{p} M \longrightarrow\{j(p)\} \times \mathbb{R}^{n}
$$

of $j$ at $p \in M$ is injective for any $p \in M$,

$$
j: M \longrightarrow j(M) \subset \mathbb{R}^{n}
$$

is a homeomorphism.

We point out at this occasion that the tangent map

$$
T j: T M \longrightarrow T \mathbb{R}^{n}=\mathbb{R}^{n} \times \mathbb{R}^{n}
$$

splits naturally into

$$
T j=\underset{(j, d j)}{\vdots}
$$

where

$$
\mathrm{dj}=p r_{2} \circ \mathrm{Tj}
$$

and

$$
\text { (1.i) } \quad \mathrm{pr}_{2}: \mathbb{R}^{\mathrm{n}} \times \mathbb{R}^{\mathrm{n}} \longrightarrow \mathbb{R}^{\mathrm{n}}
$$

is the projection onto the second factor along the first one, i.e.

$$
\operatorname{pr}_{2}(a, b)=b \quad \text { for all pairs }(a, b) \in \mathbb{R}^{n} \times \mathbb{R}^{n} \text {. }
$$

Thus dj represents locally nothing else but the Frechet differential of the local representative of $j$.

We denote by $E\left(M, \mathbb{R}^{n}\right)$ the set of all smooth embeddings of $M$ into $\mathbb{R}^{n}$. Hence $E\left(M, R^{n}\right)$ is the set of all configurations of our moving deformable medium. If we equip $E\left(M, \mathbb{R}^{n}\right)$ with the Whitney $C^{\infty}$ - topology (cf. [Gui,Go]), then it becomes an infinite dimensional Frechet manifold.

The reason is the following one :

Consider the set $C^{\infty}\left(M, \mathbb{R}^{n}\right)$ of all smooth maps of $M$ into $\mathbb{R}^{n}$, which is endowed 
with Whitney's $C^{\infty}-$ topology and note that together with the pointwise defined operations of addition and multiplication with scalars $C^{\infty}\left(M, \mathbb{R}^{n}\right)$ becomes a complete metrizable, locally convex space, a so-called Frechet space.

Since as shown in [Hi] $E\left(M, \mathbb{R}^{n}\right)$ is open in $C^{\infty}\left(M, \mathbb{R}^{n}\right)$, it hence carries the structure of a Frechet manifold (cf. [Bi,Fi]).

using now the differential calculus in locally convex spaces constructed either in $[\mathrm{Ba}],[\mathrm{Gu}],[\mathrm{Mi}]$ or $[\mathrm{Fr}, \mathrm{Kr}]$, it is evident that the tangent space $T j E\left(M, \mathbb{R}^{n}\right)$ at $j \in E\left(M, \mathbb{R}^{n}\right)$ is nothing else but $C^{\infty}\left(M, \mathbb{R}^{n}\right)$.

Therefore the tangent bundle $T E\left(M, \mathbb{R}^{n}\right)$ is trivial, i.e.

$$
T E\left(M, \mathbb{R}^{n}\right)=E\left(M, \mathbb{R}^{n}\right) \times C^{\infty}\left(M, \mathbb{R}^{n}\right)
$$

We note that $T E\left(M, \mathbb{R}^{n}\right)$ is the phase space of motions in $E\left(M, \mathbb{R}^{n}\right)$.

Next we introduce the principal bundle structure of $E\left(M, \mathbb{R}^{n}\right)$, which is crucial for our formalism to describe the motion of the deformable medium $M$. Following [Bi,Fi] we first describe the group action. Let Diff $H$ be the group of all smooth diffeomorphisms of $M$ equipped with the $C^{\infty}-$ topology. Diff $M$ is a Frechet manifold, in which the operations are smooth in either one of the above mentioned notions of differentiability.

Consequently we call Diff $M$ a differentiable group. The tangent space at the identity in Diff $\mathrm{M}$ is naturally identified with $\Gamma$ TM, the set of smooth vector fields on $M$.

The operation $\Phi$ of Diff $M$ on $E\left(M, \mathbb{R}^{\mathrm{n}}\right)$ given by

$$
\Phi(j, g)=j \circ g
$$

$\forall j \in E\left(M, \mathbb{R}^{\mathrm{n}}\right), g \in$ Diff $M$,

is smooth.

Consequently $E\left(M, \mathbb{R}^{n}\right)$ can be represented as

$$
E\left(W, \mathbb{R}^{n}\right)=\underset{i \in E\left(M, \mathbb{R}^{n}\right)}{U} \text { i o Diff } W
$$

i.e. as the collection of smooth fibers. The quotient $U\left(M, \mathbb{R}^{n}\right)$ of $E\left(M, \mathbb{R}^{n}\right)$ by Diff $M$

$$
U\left(M, \mathbb{R}^{n}\right)=E\left(M, \mathbb{R}^{n}\right) / \text { Diff } M
$$

inherits a smooth Frechet manifold and it is naturally identified with the collection of all smooth submanifols of $M$ in $\mathbb{R}^{n}$ which are diffeomorphic to $M$. Let us denote the quotient map, i.e. the projection of $E\left(M, \mathbb{R}^{n}\right)$ onto $U\left(M, \mathbb{R}^{n}\right)$, by $\Pi$. The quadruple $\left(E\left(M, \mathbb{R}^{n}\right), \Pi, U\left(M, \mathbb{R}^{n}\right)\right.$, Diff $\left.M\right)$ is then a principal bundle in the sense of $[\mathrm{Gr}, \mathrm{H}, \mathrm{V}]$.

Each configuration $j \in E\left(M, \mathbb{R}^{n}\right)$ induces a Riemannian metric $m(j)$ on $M$ defined 
by

$$
m(j)(X, Y):=\langle\operatorname{dj} X, d j Y\rangle \quad \forall X, Y \in \Gamma T M .
$$

Denoting now by $\mathfrak{M}(\mathrm{M})$ the set of all smooth Riemannian metrics on $M$ equipped with the $C^{\infty}$ - topology, we obtain then a natural map

$$
m: E\left(M, \mathbb{R}^{n}\right) \longrightarrow \mathfrak{X}_{(}(M)
$$

Noting that $\mathfrak{M}(M)$ is an open cone in $S^{2}(M)$, the Frechet space of smooth symmetric two tensors on $M$, we deduce that $\mathfrak{M}(M)$ is also a Frechet manifold and that the map $\mathrm{m}$ is smooth (cf. [Sch]).

Horeover the tangent bundle $T \mathfrak{M}(M)$ of $\mathfrak{M}(\mathrm{M})$ is trivial, i.e.

$$
T \mathfrak{M}(M)=\mathbb{M}(M) \times S^{2}(M) \text {. }
$$

For later use we calculate at this point the derivative $\operatorname{Dm}(j)(k)$ of $m$ at $j \in$ $E\left(M, \mathbb{R}^{n}\right)$ in the direction of $h \in C^{\infty}\left(M, \mathbb{R}^{n}\right)$. Due to the $\mathbb{R}$ - bilinearity of $m$ in the variable $j \in E\left(M, \mathbb{R}^{\mathfrak{n}}\right)$ we have

$$
\begin{gathered}
\operatorname{Dm}(j)(h)(X, Y)=\langle d j X, d h Y\rangle+\langle d h X, d j Y\rangle, \\
\forall X, Y \in \Gamma T M .
\end{gathered}
$$

Splitting $\mathrm{h}$ into

$$
h=d j X_{h}+h^{1}
$$

with $X_{h} \in \Gamma T M$ and $h^{\perp}$ being pointwise normal to $j(M)$ in $\mathbb{R}^{n}$, equation (1.16) turns into

$$
\begin{aligned}
\operatorname{Dm}(j)(h)(K, Y)= & \left\langle d j X, d\left(d j K_{h}\right)(Y)\right\rangle+\left\langle d\left(d j K_{h}\right), d j Y\right\rangle \\
& +\left\langle d j X, d h^{\perp} Y\right\rangle+\left\langle d h^{\perp} X, d j Y\right\rangle .
\end{aligned}
$$

Since $d\left(d j X_{h}\right) Y=d j \nabla(j)_{Y} X_{h}+S(i)\left(X_{h}, Y\right)$, where $\nabla(i)$ is the Levi-Civita connection of $\mathrm{m}(j),(1.17)$ turns into

$$
\begin{aligned}
\operatorname{Dm}(j)(h)(X, Y)= & m(j)\left(X, \nabla(j)_{Y} X_{h}\right)+m(j)\left(\nabla(j)_{X} X_{h}, Y\right\rangle \\
& +\left\langle d j X, d^{\perp} Y\right\rangle+\left\langle d h^{\perp} X, d j Y\right\rangle \\
= & L_{X_{h}}(m(j))(X, Y)+\left\langle d j X, d^{\perp} Y\right\rangle+\left\langle d h^{\perp} X, d j X\right\rangle,
\end{aligned}
$$

with $L_{X_{h}}(m(j))$ being the Lie derivative of $m(j)$.

In case $h^{\perp}=\Theta_{h} \cdot N(j)$ then

$$
\operatorname{Dm}(j)(h)(X, Y)=L_{X_{h}}(m(j))(X, Y)+2 \cdot \Theta_{h} \cdot h(j)(X, Y),
$$

with $h(j)$ the second fundamental form of $j$ defined by

$$
h(j)(X, Y):=m(j)(W(j) K, Y)=\langle d N(j) X, d j Y\rangle .
$$


Here $W(j)$ denotes the Weingarten map of $j$ given by
(1.20)
$d j w(j) X=d N(j) X$,
$\forall X \in \Gamma T M$

If $H(j)$ denotes $\operatorname{tr} W(j)$, then

(1.21)

$\operatorname{tr} \operatorname{Dm}(j)(h)(X, Y)=2 \cdot \operatorname{div}_{j} X_{h}+\Theta_{h} \cdot H(j)$

By div ${ }_{j} \chi_{h}$ we denote the divergence of $X_{h}$ formed with respect to $m(j)$. This means,

$$
\operatorname{div}_{\mathrm{j}} X_{\mathrm{h}}=\operatorname{tr} \nabla(\mathrm{j}) X_{\mathrm{h}}
$$

The function $\frac{\mathrm{H}(j)}{\mathrm{dim} M}$ is called the mean curvature of $j$, while $H(j)$ denotes the unnormalized mean curvature. 
In order to define a metric on $E\left(M, \mathbb{R}^{n}\right)$, which is adapted to the mass distribution of our moving deformable medium $M$, we first introduce a density map

$$
\rho: E\left(M, \mathbb{R}^{n}\right) \longrightarrow C^{\infty}(M, \mathbb{R})
$$

which is supposed to be smooth in either sense of the above mentioned notions of differentiability. In addition we require that $\rho$ fullfills a continuity equation, namely

$$
D \rho(j)(h)=-\frac{1}{2} \rho(j) \operatorname{tr}_{j} D m(j)(h), \quad \forall j \in E\left(M, \mathbb{R}^{n}\right), h \in C^{\infty}\left(M, \mathbb{R}^{n}\right),
$$

where $\operatorname{tr} r_{j}$ denotes the trace taken with respect to $m(j)$. Lsing the fact that the derivative at $j$ in the direction of any $h \in C^{\infty}\left(M, R^{n}\right)$ of the Riemannian volume form $\mu(j)$ has the form

$$
D \mu(j)(h)=\frac{1}{2} \mu(j) \operatorname{tr}_{j} D m(j)(h)
$$

as shown in $[\mathrm{Bi}, 1]$, it follows that $(2.2)$ is indeed the continuity equation. Consequently the total mass $m(j)$ attached to any $j \in E\left(M, R^{n}\right)$ via the formula

$$
m(j)={ }_{M} \rho(j) \mu(j)
$$

is constant in $\mathrm{j}$.

The existence of such a function $\rho$ can be established as follows:

Let $i \in E\left(M, \mathbb{R}^{n}\right)$ be any embedding and denote by $O_{i}$ its connected component in $E\left(M, \mathbb{R}^{n}\right)$. Then for any $j \in O_{i}$ the differential $d j$ is related to di in the following way

$$
d j=g \circ d i \circ f,
$$

where $g \in C^{\infty}(M, S O(n))$ and $f$ is a strong bundle isomorphism of $T$ Wh which is fibrewise positive with respect to $m(i)$. One easily verifies that the Riemannian volume forms $\mu(j)$ and $\mu(i)$ of $j$ and i respectivel $\because$ are related by

$$
\mu(j)=\operatorname{det} f \cdot \mu(i)
$$

We then set

$$
\rho(j):=\rho(i) \cdot \operatorname{det} f^{-1}
$$

with $\rho(i)$ chosen such that

$$
\rho(\mathrm{i})(\mathrm{p})>0, \quad \forall p \in \mathrm{M} \text {, }
$$


and note that this map satisfies the continuity equation (2.2).

Next we introduce the metric $\mathfrak{B}_{E}$ on $E\left(M, \mathbb{R}^{n}\right)$ by the formula

$$
\mathfrak{B}_{E}(j)(h, k):=W \int \rho(j)\langle h, k\rangle \mu(j), \quad \forall h, k \in C^{\infty}\left(M, R^{n}\right)
$$

Due to $(2.2) \mathbb{B}_{E}$ is constant in $j$. Therefore the geodesics of $\mathbb{B}_{E}$ are straight line segments as shown in $[B i, 1]$. 
By a constitutive law we understand a smooth parameter depending one form, the so-called work

$$
F: C^{\infty}\left(M, \mathbb{R}^{n}\right) \times E\left(M, \mathbb{R}^{n}\right) \times C^{\infty}\left(M, \mathbb{R}^{n}\right) \longrightarrow \mathbb{R}
$$

The first factor $C^{\infty}\left(M, \mathbb{R}^{n}\right)$ in the above cartesian product is the parameter space. We often will regard $F$ as a map

$$
F: C^{\infty}\left(M, \mathbb{R}^{n}\right) \longrightarrow A^{1}\left(E\left(M, \mathbb{R}^{n}\right), \mathbb{R}\right)
$$

The domain of $F$ is the parameter space, the range the collection of all smooth one forms on $E\left(M, \mathbb{R}^{n}\right)$ with values in $\mathbb{R}$. To handle this abstract notion (3.1) we require an integral representation for $F$ given by

$$
F(k)(j, h)={ }_{M} \int\left\langle\varphi_{F}(j, k), h\right\rangle \mu(j), \quad \forall j \in E\left(M, \mathbb{R}^{n}\right), h, k \in C^{\infty}\left(M, \mathbb{R}^{n}\right),
$$

where $P_{F}: T E\left(M, \mathbb{R}^{n}\right) \rightarrow C^{\infty}\left(M, \mathbb{R}^{n}\right)$, the so-called force density, is assumed to be a smooth map. We point out that $F(k)(j, h)$ varies linearly only in $h$ and that it furthermore depends on the maps $j$ and $k$ globally.

The equation of motion on $E\left(M, \mathbb{R}^{n}\right)$ described by a smooth curve

$$
\sigma:(-\lambda, \lambda) \longrightarrow E\left(M, \mathbb{R}^{\mathrm{n}}\right)
$$

for some positive real $\lambda$, is given by

$$
\begin{gathered}
F(\dot{\sigma}(t))(\sigma(t), h)=B(j)(\tilde{\sigma}(t), h)={ }_{M} \int\left\langle P_{F}(\sigma(t), \dot{\sigma}(t)), h\right\rangle \mu(\sigma(t)) \\
={ }_{M} \int \rho(\sigma(t))\langle\ddot{\sigma}(t), h\rangle \mu(\sigma(t)) \begin{array}{c} 
\\
\forall h \in C^{\infty}\left(M, \mathbb{R}^{n}\right),
\end{array}
\end{gathered}
$$

where for the sake of simplicity we have taken $k=\dot{\sigma}(t)$.

It is obvious that this equation implies

$$
\rho(\sigma(t)) \ddot{\sigma}(t)=\varphi_{F}(\sigma(t), \dot{\sigma}(t)), \quad \forall t \epsilon(-\lambda, \lambda)
$$

We note that $(3.5)$ is a second order differential equation on $E\left(M, \mathbb{R}^{n}\right)$ and not on parts of $\mathbb{R}^{n}$.

We rewrite it now according to the principal bundle structure of $E\left(M, \mathbb{R}^{n}\right)$, by proceeding as follows :

At first we note that $\dot{\sigma}(t)$ admits in $\mathbb{R}^{\mathrm{n}}$ the pointwise splitting

$$
\dot{\sigma}(t)=\mathrm{d} \sigma(t) Z(t)+\dot{\sigma}(t)^{\perp}
$$

where $Z(t) \in \Gamma T M$ is uniquely determined and $\dot{\sigma}(t)^{\perp}$ is, according to the definition, pointwise perpendicular to $\sigma(t)(\mathrm{M})$ for each $t \in(-\lambda, \lambda)$.

Consequently $\ddot{\sigma}(t)$ is given by 


$$
\ddot{\sigma}(t)=d \dot{\sigma}(t) Z(t)+d \sigma(t) \dot{Z}(t)+\left(\dot{\sigma}(t)^{\perp}\right)^{\prime}, \quad t \in(-\lambda, \lambda)
$$

where

$$
\begin{aligned}
d \dot{\sigma}(t) Z(t)= & d(d \sigma(t) Z(t)) Z(t)+d \dot{\sigma}(t)^{\perp} Z(t) \\
= & d \sigma(t) \nabla(\sigma(t)) Z(t) Z(t)+S(\sigma(t))(Z(t), Z(t)) \\
& +d \sigma(t) W\left(\sigma(t), \dot{\sigma}(t)^{\perp}\right) Z(t)+\left(d \dot{\sigma}(t)^{\perp} Z(t)\right)^{\perp}
\end{aligned}
$$

Here $\nabla(\sigma(t))$ means the Levi - Civita connection of $m(\sigma(t))$, and $W(\sigma(t), \dot{\sigma}(t))$, the Weingarten map of $\sigma(t)$ is defined as follows :

Let $N: M \longrightarrow \mathbb{R}^{n}$ be any vector field along $j \in E\left(M, \mathbb{R}^{n}\right)$, such that

$$
\langle d j X(p), N(p)\rangle=0, \quad \forall X \in \Gamma T M, p \in M
$$

Then the Weingarten map $W(j, N)$ of $j$ given by $N$ is the uniquely determined bundle map of TM for which

$$
\operatorname{dj} W(j, N) Y=(d N Y)^{T} \text {. }
$$

In the particular case where $\mathrm{N}$ is oriented and of dimension equal to $\mathrm{n}-1$ and $N$ coincides with the unit normal vector field $N(j)$ of $j(M)$ in $\mathbb{R}^{n}$, then $W(j, N(j))$ is nothing else but the Weingarten map, denoted in this particular case just by $W(j)$.

Let us turn back to $\ddot{\sigma}(t)$. Obviously (compare cf. $[B i, 6]$ )

$$
\begin{aligned}
: \ddot{\sigma}(t)= & d \sigma(t) \nabla(\sigma(t))_{Z(t)^{Z}(t)+d \sigma(t) W\left(\sigma(t), \dot{\sigma}(t)^{\perp}\right) Z(t)} \\
& +\left(\left(\dot{\sigma}(t)^{\perp}\right)^{\prime}\right)^{\mathrm{L}}+d \sigma(t) Z(t)+S(\sigma(t))(Z(t), Z(t)) \\
& +\left(d \dot{\sigma}(t)^{\perp} Z(t)\right)^{\perp}+\left(\left(\dot{\sigma}(t)^{\perp}\right)^{\perp}\right)^{\perp} .
\end{aligned}
$$

For unifying terminology we set

$$
\left(\left(\dot{\sigma}(\mathrm{t})^{\perp}\right)^{\prime}\right)^{\mathrm{T}}:=\mathrm{d} \sigma(\mathrm{t}) \mathrm{U}(\sigma(\mathrm{t}), \dot{\sigma}(\mathrm{t}))^{\gamma}
$$

for each $t \in(-\lambda, \lambda)$ and a well defined vector field $i(\sigma(t), \dot{\sigma}(t))$ E TTM. If we split now $P_{F}(\sigma(t), \dot{\sigma}(t))$ into a tangential and normal part respectively then for all $t \in(-\lambda, \lambda)$

$$
\begin{aligned}
\varphi_{F}(\sigma(t), \dot{\sigma}(t)) & =\varphi_{F}(\sigma(t), \dot{\sigma}(t))^{T}+\varphi_{F}(\sigma(t), \dot{\sigma}(t))^{\perp} \\
& =d \sigma(t) Y_{F}(\sigma(t), \dot{\sigma}(t))+\varphi_{F}(\sigma(t), \dot{\sigma}(t))^{\perp}
\end{aligned}
$$

holds for a uniquely determined $Y_{F}(\sigma(t), \dot{\sigma}(t)) \in \Gamma T M$.

The equation of motion splits thus into the coupled system

$$
\begin{aligned}
\nabla(\sigma(t))_{Z(t)} Z(t)+ & \dot{Z}(t)+W\left(\sigma(t), \dot{\sigma}(t)^{\perp}\right) Z(t)+U(\sigma(t), \dot{\sigma}(t)) \\
= & \rho^{-1}(\sigma(t)) Y_{F}^{\prime}(\sigma(t), \dot{\sigma}(t)), \\
\left(\left(\dot{\sigma}(t)^{\perp}\right)^{\perp}\right)^{\perp}= & \rho^{-1}(\sigma(t)) \varphi_{F}(\sigma(t), \dot{\sigma}(t))^{\perp} \\
& -\left(d \dot{\sigma}(t)^{\perp} Z(t)\right)^{\perp}-S(\sigma(t))(Z(t), Z(t)) .
\end{aligned}
$$


The first equation multiplied on both sides by $\mathrm{d} \sigma(\mathrm{t})$ yields an equation of vectors which are tangential to $\sigma(t)$ o Diff $M$, while the second one is an equation of vectors in $C^{\infty}\left(M, \mathbb{R}^{n}\right)$, which are normal to $\sigma(t) \circ$ Diff $M$.

Hence the above coupled system (3.14) is a splitting of the equation of motion according to the principal bundle structure of $E\left(M, \mathbb{R}^{\mathrm{n}}\right)$ as mentioned above.

In the particular case $\operatorname{dim} M=n-1$ we obtain

$$
\begin{aligned}
& \dot{\sigma}(t)^{\perp}=\epsilon(\sigma(t), \dot{\sigma}(t)) N(\sigma(t)) \\
& \varphi_{F}(\sigma(t), \dot{\sigma}(t))^{\perp}=k_{F}(\sigma(t), \dot{\sigma}(t)) N(\sigma(t))
\end{aligned}
$$

for well determined $\epsilon(\sigma(t), \dot{\sigma}(t)), \kappa_{F}(\sigma(t), \dot{\sigma}(t)) \in C^{\infty}(\mathbb{M}, \mathbb{R})$.

Hence

$$
\left(\dot{\sigma}(t)^{\perp}\right)^{\cdot}=\dot{\epsilon}(\sigma(t), \dot{\sigma}(t)) N(\sigma(t))+\epsilon(\sigma(t), \dot{\sigma}(t)) N(\sigma(t))^{\prime}
$$

It remains now to calculate $N(\sigma(t))^{\prime}$. To this end we prove at first that

$$
\operatorname{DN}(j)(\tau \cdot N(j))=-d j \operatorname{grad}_{j} \tau, \quad \forall \tau \in C^{\infty}(M, \mathbb{R})
$$

Indeed let

$$
j(t)=j+t \cdot \tau \cdot N(j), \quad \forall t \in \mathbb{R} .
$$

Then we get

$$
\begin{aligned}
\frac{d}{d t} & \left.\langle d j(t), N(j(t))\rangle\right|_{t=0}=0 \\
& =\langle\tau \cdot d j W(j), N(j)\rangle+d \tau+\langle d j, D N(j)(\tau \cdot N(j))\rangle .
\end{aligned}
$$

Using that $\langle N(j), N(j)\rangle=1,(3.18)$ then follows.

Next consider a smooth curve $\gamma(t) \in$ Diff $M, t \in(-\lambda, \lambda), \lambda>0$, and note that

$$
N(j \circ \gamma(t))=N(j) \circ \gamma(t)
$$

Differentiating $(3.20)$ we get

$$
\operatorname{DN}(j)(d j X)=\operatorname{dN}(j) X=d j W(j) X,
$$

where $x=\dot{\gamma}(0)$. From $(3.6),(3.15),(3.18)$ and (3.21) it follows that

$$
\begin{aligned}
N(\sigma(t))^{\prime} & =D N(\sigma(t)) N(d \sigma(t) Z(t)+\epsilon(\sigma(t), \dot{\sigma}(t)) N(\sigma(t)) \\
& =d \sigma(t)\left(W(\sigma(t)) Z(t)-\operatorname{grad}_{\sigma(t)} \epsilon(\sigma(t), \dot{\sigma}(t))\right),
\end{aligned}
$$

and hence
$\left(3.23_{1}\right)$
$\mathrm{U}(\sigma(\mathrm{t}), \dot{\sigma}(\mathrm{t}))=\epsilon(\sigma(\mathrm{t}), \dot{\sigma}(\mathrm{t})) \mathrm{W}(\sigma(\mathrm{t})) Z(\mathrm{t})-\operatorname{grad}_{\sigma(\mathrm{t})} \epsilon(\sigma(\mathrm{t}), \dot{\sigma}(\mathrm{t}))$
$\left(3.23_{2}\right)$
$\left(\left(\dot{\sigma}(t)^{\perp}\right)^{\prime}\right)^{\perp}=\dot{\epsilon}(\sigma(t), \dot{\sigma}(t)) N(\sigma(t))$. 
Moreover

$$
\begin{aligned}
\mathrm{d}\left(\dot{\sigma}(t)^{\perp}\right) Z(t)= & \mathrm{d} \varepsilon(\sigma(t), \dot{\sigma}(t)) Z(t) N(\sigma(t)) \\
& +\epsilon(\sigma(t), \dot{\sigma}(t)) \mathrm{d} \sigma(t) W(\sigma(t)) Z(t) \\
& -\mathrm{d} \sigma(t) \operatorname{grad}_{\sigma(t)} \epsilon(\sigma(t), \dot{\sigma}(t)) .
\end{aligned}
$$

Thus (3.14) rewrites as

$$
\begin{gathered}
\nabla(\sigma(t))_{Z(t)} Z(t)+\dot{Z}(t)+2 \epsilon(\sigma(t), \dot{\sigma}(t)) W(\sigma(t) Z(t)) \\
-\operatorname{grad}_{\sigma(t)} \epsilon(\sigma(t), \dot{\sigma}(t))=\rho^{-1}(\sigma(t)) Y(\sigma(t), \dot{\sigma}(t)), \\
\dot{\epsilon}(\sigma(t), \dot{\sigma}(t))=\rho^{-1}(\sigma(t)) \kappa_{F}(\sigma(t), \dot{\sigma}(t))+\hat{h}(\sigma(t))(Z(t), Z(t)) \\
-d \epsilon(\sigma(t), \dot{\sigma}(t)) Z(t) .
\end{gathered}
$$

We refer to (3.25) as the general equations of motion of a deformable medium.

Let us now split $Y_{F}(\sigma(t), \dot{\sigma}(t))$ with respect to $m(\sigma(t))$ ) according to the Hodge decomposition into

$$
Y_{F}(\sigma(t), \dot{\sigma}(t))=Y_{F}^{0}(\sigma(t), \dot{\sigma}(t))+\operatorname{grad}_{\sigma(t)} \tau_{F}(\sigma(t), \dot{\sigma}(t))
$$

where $\tau(\sigma(t), \dot{\sigma}(t)) \in C^{\infty}\left(M, \mathbb{R}^{\mathrm{n}}\right)$ and

$$
\operatorname{div}_{\sigma(t)} \mathrm{Y}_{F}^{0}(\sigma(\mathrm{t}), \dot{\sigma}(\mathrm{t}))=0
$$

i.e. the divergence of $Y_{F}^{\circ}(\sigma(t), \dot{\sigma}(t))$ taken with respect to $m(\sigma(t))$ vanishes, $\tau_{F}(\sigma(t), \dot{\sigma}(t)) \in C^{\infty}(M, R)$, and $\operatorname{grad}_{\sigma(t)}$ means the gradient taken with respect to $m(\sigma(t))$.

Lising (3.26) we rewrite the first equation (3.25) as

$$
\begin{aligned}
& \nabla(\sigma(t))_{Z(t)} Z(t)+\dot{Z}(t)+2 \epsilon(\sigma(t), \dot{\sigma}(t)) W(\sigma(t) Z(t) \\
& -\operatorname{grad}_{\sigma(t)} \epsilon(\sigma(t, \dot{\sigma}(t)) \\
& =\rho^{-1}(\sigma(t))\left[\operatorname{grad}_{\sigma(t)} \tau_{F}(\sigma(t), \dot{\sigma}(t))+Y_{F}^{0}(\sigma(t), \dot{\sigma}(t))\right] .
\end{aligned}
$$

The above Hodge decomposition of $Y_{F}(\sigma(t), \dot{\sigma}(t))$ vields a decomposition of $\varphi_{F}$ into

$$
P_{F}(\sigma(t), \dot{\sigma}(t))=\varphi_{F}^{0}(\sigma(t), \dot{\sigma}(t))+\varphi_{F}^{\prime}(\sigma(t), \dot{\sigma}(t))
$$

where

$$
\left\{\begin{array}{l}
\varphi_{F}^{0}(\sigma(t), \dot{\sigma}(t))=d \sigma(t) \operatorname{grad}_{\sigma(t)} \tau_{F}(\sigma(t), \dot{\sigma}(t)), \\
\varphi_{F}^{\prime}(\sigma(t), \dot{\sigma}(t))=d \sigma(t) Y_{F}^{o}(\sigma(t), \dot{\sigma}(t))+\varphi_{F}(\sigma(t), \dot{\sigma}(t))^{\perp} .
\end{array}\right.
$$

We note that the tangential part of the force density $\varphi_{F}^{\prime}$ is divergence free. Its corresponding work, i.e. the one form $F$, will be called the reduced constitutive law. 


\section{The motion along a fixed surface $i(M) \subset \mathbb{R}^{\mathrm{n}}$}

Let us consider again the coupled system (3.14) describing the motion of the deformable medium $M$. Noting that the embedding $\sigma(t)$ varies with $t$ and that the submanifolds $\sigma\left(t_{1}\right)(M)$ and $\sigma\left(t_{2}\right)(M)$ of $\mathbb{R}^{n}$ differ generically from each other for different $t_{1}, t_{2} \in(-\lambda, \lambda)$, we obtain that the first equation (3.14) describes the instantaneous motion of the deformable medium along the submanifold $\mathrm{M}$.

In this section we assume that the submanifolds $\sigma(t)(M)$ of $\mathbb{R}^{\mathrm{n}}$ are identical for all $t \in(-\lambda, \lambda)$.

As a visualising example we image a fluid moving on a sphere of fixed radius.

More generally let $i \in E\left(M, \mathbb{R}^{n}\right)$ be fixed.

Thus $i(M)$ is a submanifold of $\mathbb{R}^{\mathrm{n}}$ on which a deformable medium moves according to a constitutive law to be specified below. A configuration of this motion is an embedding $j$ of $H$ onto $i(M)$ and is hence of the form

$$
j=i \circ g \text {, }
$$

for some $g \in$ Diff $M$.

Consequently the configuration space is $i$ o Diff $M$. It remains now to specify the constitutive law on $T(i \circ$ Diff $M)$, the phase space of the motions on $i(M)$. To this end let us first study the nature of a tangent vector $h$ to $i$ Diff $M$ at $i \circ g$, i.e. $h \in T_{i o g} i$ o Diff $M$. If we denote by $R_{g}$ the right translation by g, i.e.

$$
\left\{\begin{array}{l}
R_{\mathrm{g}}: \text { Diff } M \longrightarrow \text { Diff } M, \\
\mathrm{~g}^{\prime}, \mapsto \quad \mathrm{g} \circ \mathrm{g}^{\prime},
\end{array}\right.
$$

then the tangent map $\mathrm{TR}_{\mathrm{g}}$ (id) sends any tangent vector $X \in \Gamma T \mathrm{M}$ at Id Diff $\mathrm{M}$ into a tangent vector in $\mathrm{T}_{\mathrm{g}}$ Diff $\mathrm{M}$. Moreover

$$
\mathrm{TR}_{\mathrm{g}}: \Gamma \mathrm{TM} \longrightarrow \mathrm{T}_{\mathrm{g}} \text { Diff } \mathrm{M}
$$

is obviously surjective. Regarding $j \circ g \circ$ Diff $M$ as a submanifold of $E\left(M, \mathbb{R}^{n}\right)$, any $h \in T_{j o g} E\left(M, \mathbb{R}^{n}\right)$ tangential to $j \cap g \circ$ Diff $M$ is thus of the form

$$
\mathrm{h}=\mathrm{d}(\mathrm{i} \text { o } \mathrm{g}) \mathrm{x}_{\mathrm{h}} \text {, }
$$

for a uniquely defined vector field $X_{h} \in$ rTM.

Thus we have a natural bijection

$$
d(i \circ g): \Gamma T M \longrightarrow T_{i \circ g} i \circ \text { Diff } M \subset T_{i \circ g} E\left(M, \mathbb{R}^{n}\right),
$$


sending each $X \in \Gamma T M$ into $d(i \circ g) X \in T_{\text {iog }}$ i o Diff $M$.

By (4.5) we see that $T$ ( $i$ o Diff $M$ ) is trivialized via right translations as

$T(i \circ \operatorname{Diff} M)=i \circ \operatorname{Diff} M \ddot{x} \Gamma T M$.

Clearly

$$
d(i \circ g) X=d i \circ T_{g} X
$$

We now introduce the constitutive law via the smooth map

$$
F: \operatorname{di} \Gamma T M \times \text { i } \circ \text { Diff } M \times C^{\infty}\left(M, \mathbb{R}^{n}\right) \longrightarrow \mathbb{R},
$$

which is linear in $h \in C^{\infty}\left(M, \mathbb{R}^{n}\right)$. We note that in analogy to (3.1) we put the parameter space di $\Gamma T M$ as the first factor of the domain of definition. The justification for choosing the third factor as being $C^{\infty}\left(M, R^{n}\right)$ instead or TTM will be given later.

As in the preceding section we require an integral representation for the constitutive law reading as

$$
\begin{gathered}
F(d(i \circ g) X)(i \circ g, h)=\int\left\langle\varphi_{F}(i \circ g, d(i \circ g) X, h\rangle \mu(i \circ g)\right. \\
\forall h \in C^{\infty}\left(M, \mathbb{R}^{n}\right), X \in \Gamma T M, g \in \text { Diff } M,
\end{gathered}
$$

where the force density

$$
\varphi_{F}: \text { i o Diff } M \times \Gamma T M \longrightarrow C^{\infty}\left(M, \mathbb{R}^{n}\right)
$$

is a smooth map.

The equation of motion on i o Diff $M$ described by a smooth curve

$$
\sigma:(-\lambda, \lambda) \longrightarrow \text { i o Diff } \mathrm{M}, \quad \lambda>0,
$$

subjected to the above constitutive law is hence

$$
\rho(\sigma(\mathrm{t})) \ddot{\sigma}(\mathrm{t})=\varphi_{\mathrm{F}}(\sigma(\mathrm{t}), \dot{\sigma}(\mathrm{t})),
$$

or, equivalently,

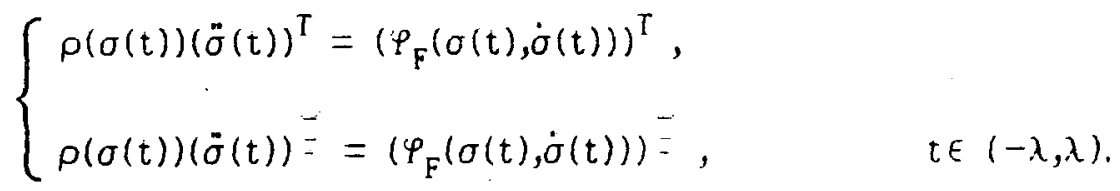

We obviously have

$$
\left(\varphi_{F}(\sigma(t), \dot{\sigma}(t))\right)^{T}=d \sigma(t) Y_{F}(\sigma(t), \ddot{\sigma}(t)),
$$

for a well defined vector field $\mathrm{Y}_{\mathrm{F}}(\sigma(t), \dot{\sigma}(\mathrm{t})) \in \Gamma T M$. Since a solution $\sigma(t)$ of (4.13) has the form 
for a smooth map

$$
g:(-\lambda, \lambda) \longrightarrow \text { Diff } \mathrm{M}
$$

it follows that

$$
\begin{aligned}
\dot{\sigma}(t) & =d(\dot{i} \circ g(t)) Z(t) \\
& =d i \circ T_{g}(t) Z(t),
\end{aligned}
$$

where $Z(t) \in \Gamma T M$ is well determined, and consequently

$$
\begin{aligned}
\ddot{\sigma}(t)= & (d(i \circ g(t)) Z(t)) \\
= & d(i \circ g(t)) Z(t)+d(i \circ g(t)) Z(t) \\
= & d(i \circ g(t)) \nabla(i \circ g(t)) Z(t) Z(t)+ \\
& d(i \circ g(t)) Z(t)+s(i \circ g(t))(Z(t), Z(t)) .
\end{aligned}
$$

Using (4.1T), from (4.13) we obtain, $\forall t \in(-\lambda, \lambda)$,

$$
\left\{\begin{array}{l}
\rho(i \circ g(t))(\nabla(i \circ g(t)) Z(t) Z(t)+Z(t))=Y_{F}(g(t), Z(t)), \\
\rho(i \circ g(t)) S(i \circ g(t))(Z(t), Z(t))=\varphi_{F}(g(t), Z(t))^{\perp},
\end{array}\right.
$$

where we have used the notation

$$
f(g(t), Z(t)):=f(i \circ g(t), d(i \circ g(t)) Z(t)) \text {. }
$$

By comparing (4.18) with (3.14) we observe that the last equations are obtained from the first ones by setting $\dot{\sigma}(t)^{\perp}=0$, in accordance with the fact that $i(M)$ is a fixed surface.

We note that we can remove the instantaneous connection $\nabla(\mathrm{i} \circ \mathrm{g}(\mathrm{t}))$ and the instantaneous second fundamental tensor $S(\mathrm{i}$ o $\mathrm{g}(\mathrm{t}))$ in $(4.19)$ by using the push-forward of $Z(t)$ by $g(t) \in$ Diff $M$, that is we introduce $Z(t)$ b:

$$
X(t):=\operatorname{Tg}(t) Z(t) g(t)^{-1}, \quad \quad t \in(-\lambda, \lambda) .
$$

Using (4.20) we obtain on one hand

$$
\left\{\begin{array}{l}
\dot{\sigma}(t)=d i X(t) g(t)=d(i \circ g(t)) Z(t), \\
\ddot{\sigma}(t)=d i X(t) g(t)+d(d i X(t)) g(t) .
\end{array}\right.
$$

On the other hand the equation

$$
\dot{\sigma}(t)=d i \dot{g}(t)=d i X(t) g(t)
$$

yields

$$
\dot{g}(t)=X(t) g(t) .
$$


(4.21) and (4.23) imply

$$
\ddot{\sigma}(t) g(t)^{-1}=d^{\prime} X(t)+d i \nabla(i)_{X(t)} X(t)+S(i)(X(t), X(t)) .
$$

Setting now

$$
\left\{\begin{array}{l}
Y_{F}(g(t), X(t)):=Y_{F}(g(t), Z(t)) g(t), \\
P_{F}(g(t), X(t))^{\perp}:=\left(\varphi_{F}(g(t), Z(t))\right)^{\perp} g(t), \quad t \in(-\lambda, \lambda)
\end{array}\right.
$$

and observing that the map

$$
\rho: E\left(M, \mathbb{R}^{n}\right) \longrightarrow C^{\infty}(M, \mathbb{R})
$$

does depend by construction on $d j$ rather than on $j$ itself the system (4.18) turns into

$$
\left\{\begin{array}{l}
\left.\rho(d \sigma(t))(\nabla(\mathrm{i}))_{X(\mathrm{t})} X(\mathrm{t})+X(\mathrm{t})\right)=Y_{F}(g(t), X(\mathrm{t})), \\
\rho(\mathrm{d} \sigma(\mathrm{t})) S(\mathrm{i})(X(\mathrm{t}), X(\mathrm{t}))=\varphi_{F}(g(t), X(\mathrm{t}))^{\perp} .
\end{array}\right.
$$

Let us note that in the case when we would require that $F(d(i \circ g))$ would act on $\Gamma T M$ rather than on $C^{\infty}\left(M, \mathbb{R}^{n}\right)$, we would obtain only the equations (4.13), by missing the observation that the normal forces are up to the density $\rho$ of a geometric nature.

The next step is to decompose $Y_{F}(g(t), X(t))$ with respect to $m(i \circ g(t))$ according to Hodge uniquely into

$(t .2 \tau) \quad\left\{\begin{array}{l}Y_{F}(g(t), X(t))=\operatorname{grad}_{i} \tau_{F}(g(t), Z(t))+Y_{F}^{0}(g(t), Z(t)), \\ \operatorname{dir}_{i} r_{F}^{0.0}(g(t), Z(t))=0,\end{array}\right.$

where $T_{F}(g(t), X(t)) \in C^{\infty}(M, \mathbb{R})$.

Thus the first equation $(4.26)$ becomes

$\left.(1.26)^{\prime}\right)$

$$
\begin{aligned}
& \rho(X(t))(\nabla(i) X(t) X(t)+X(t)) \\
& =\operatorname{grad}_{i} \tau_{F}(g(t), X(t))+Y^{0}(g(t), X(t)) .
\end{aligned}
$$

If we require

$$
\sigma((-\lambda, \lambda)) \subset \mathrm{i} \circ \operatorname{Diff}_{\mu(\mathrm{i})} \text { H, }
$$

where Diff $\operatorname{Di}_{\mu} \mathrm{M}$ is the subgroup of all elements in Diff $\mathrm{H}$ which leave $\mu(i)$ invariant, then $X(t)$ has to be divergence free for all $t \in(-\lambda, \lambda)$.

This is due to the fact that 
(4.29)

$$
T_{\text {id }} \text { Diff } M=\left\{X \in \Gamma T M \mid \operatorname{div}_{i} X=0\right\}
$$

In this case $\sigma(t)$ has to satisfy the system of equations

$(4.30)$

$$
\left\{\begin{array}{l}
\rho(\nabla(i) X(t) X(t)+X(t)) \\
\quad=\operatorname{grad}_{i} \tau_{F}(g(t), X(t))+Y_{F}^{0}(g(t), X(t)), \\
\rho S(i)(X(t), X(t))=\varphi_{F}(\sigma(t), X(t))^{\perp}, \\
\operatorname{div}_{i} X(t)=0,
\end{array}\right.
$$

with $\rho:$ i a Diff $M \longrightarrow \mathbb{R}$ being a constant furction. 
Let us assume now that the motion of the deformable medium $M$ is subjected to an internal constitution law $\mathrm{F}$, which admits an integral representation.

The fact that the corresponding force density is an internal one requires i $i$ to be independent of the region in $\mathbb{R}^{n}$ in which the deformable medium moves. Hence an internal force density has to be invariant under the translation or, more precisely, under the action of the translation group $\mathbb{R}^{\mathbf{n}}$ of $\mathbb{R}^{\mathbf{n}}$ (cf. $[\mathrm{Bi}, 4]$ ).

Let us describe next this action of $\mathbb{R}^{n}$ on $T E\left(M, \mathbb{R}^{n}\right)$.

At first we recall that the translation group $\mathbb{R}^{\mathrm{n}}$ of the vector space $\mathbb{R}^{\mathrm{n}}$ is the underlying abelian group of the $\mathbb{R}$-vector space $\mathbb{R}^{\mathrm{n}}$.

The action

$$
r: C^{\infty}\left(M, \mathbb{R}^{n}\right) \times \mathbb{R}^{n} \longrightarrow C^{\infty}\left(M, \mathbb{R}^{n}\right)
$$

on $C^{\infty}\left(M, \mathbb{R}^{n}\right)$ is given by

$$
r(h, z)=h+z, \quad \forall h \in C^{\infty}\left(M, \mathbb{R}^{n}\right), z \in \mathbb{R}^{n},
$$

where by $h+z$ we mean the map defined via

$$
(h+z)(p)=h(p)+z, \quad \forall p \in M .
$$

Hence $z \in \mathbb{R}^{\mathrm{n}}$ is naturally identified with the constant map in $C^{\infty}\left(M, \mathbb{R}^{\mathrm{n}}\right)$ assuming $z$ as its value.

Clearly in the particular case where $h=j \in E\left(M, \mathbb{R}^{n}\right)$

$$
r(j, z)=j+z, \quad \forall z \in E\left(M, \mathbb{R}^{n}\right),
$$

belongs to $E\left(M, \mathbb{R}^{n}\right)$. Hence $r$ reduces to

$$
r: E\left(M, \mathbb{R}^{n}\right) \times \mathbb{R}^{n} \longrightarrow E\left(M, \mathbb{R}^{n}\right)
$$

The tangent map $\operatorname{Tr}$ of $r$ is given by

$$
\begin{aligned}
\operatorname{Tr}(h, z)(k, u)= & (h+z, k+u), \\
& \forall k \in T_{h} C^{\infty}\left(M, \mathbb{R}^{n}\right), h \in C^{\infty}\left(M, \mathbb{R}^{n}\right), z, u \in \mathbb{R}^{n} .
\end{aligned}
$$

Hence $r$ induces an action on $T C^{\infty}\left(M, \mathbb{R}^{n}\right)$ defined by

$$
\begin{aligned}
\operatorname{Tr}: & \operatorname{TC}^{\infty}\left(M, \mathbb{R}^{n}\right) \times T \mathbb{R}^{n} \longrightarrow T C^{\infty}\left(M, \mathbb{R}^{n}\right), \\
((h, k),(z, u)) & \mapsto(h+z, k+u),
\end{aligned}
$$

where $k \in T_{h} C^{\infty}\left(M, \mathbb{R}^{n}\right)=C^{\infty}\left(M, \mathbb{R}^{n}\right)$ and $u \in T_{z} \mathbb{R}^{n}=\mathbb{R}^{n}$, and respectively on $T E\left(M, \mathbb{R}^{n}\right)$ given by

$$
\begin{gathered}
\operatorname{Tr}: \operatorname{TE}\left(M, \mathbb{R}^{\mathrm{n}}\right) \times \operatorname{TR}^{\mathrm{n}} \longrightarrow \operatorname{TE}\left(M, \mathbb{R}^{\mathrm{n}}\right), \\
((j, k),(z, u)) \mapsto(j+z, k+u),
\end{gathered}
$$


where $k \in T_{j} E\left(M, \mathbb{R}^{n}\right)=C^{\infty}\left(M, \mathbb{R}^{n}\right)$ and $u \in T_{z} \mathbb{R}^{n}=\mathbb{R}^{n}$.

Given now a parameter depending smooth constitutive law

$$
F: C^{\infty}\left(M, \mathbb{R}^{n}\right) \longrightarrow A^{1}\left(E\left(M, \mathbb{R}^{n}\right), \mathbb{R}\right)
$$

and continuing to write

$$
F(j, k) \text { instead of } F(k, j), j \in E\left(M, \mathbb{R}^{n}\right), k \in C^{\infty}\left(M, \mathbb{R}^{n}\right)
$$

we form next

$$
F \circ \operatorname{Tr}((j, k),(z, u)): C^{\infty}\left(M, \mathbb{R}^{n}\right)=T_{j+z} E\left(M, \mathbb{R}^{n}\right) \longrightarrow \mathbb{R}
$$

The requirement

$$
\begin{aligned}
F(j+z, & k+u)=F(j, k) \\
& \forall j \in E\left(M, \mathbb{R}^{n}\right), k \in C^{\infty}\left(M, \mathbb{R}^{n}\right), z, u \in \mathbb{R}^{n},
\end{aligned}
$$

does then yield the type of constitutive law we want to work with. In order to construct the desired type of $\mathbb{R}^{\mathrm{n}}$-invariant constitutive laws, we consider the quotients of the actions $r$ and $T r$. To this end we note that the map

$$
\mathrm{d}: \mathrm{C}^{\infty}\left(\mathrm{M}, \mathbb{R}^{\mathrm{n}}\right) \longrightarrow\left\{\mathrm{dh} \mid \mathrm{h} \in \mathrm{C}^{\infty}\left(\mathrm{M}, \mathbb{R}^{\mathrm{n}}\right)\right\}
$$

has the property that

$$
\mathrm{d}^{-1}(\mathrm{~d} h)=\{\mathrm{h}+\mathrm{z} \mid z \in \mathbb{R}\} .
$$

Hence if we quotient out the action of $\mathbb{R}^{n}$ on $C^{\infty}\left(M, \mathbb{R}^{n}\right)$ we obtain a bijection again called $d$

$$
d:\left.C^{\infty}\left(M, \mathbb{R}^{n}\right)\right|_{\mathbb{R}^{n}} \longrightarrow\left\{d h \mid h \in C^{\infty}\left(M, \mathbb{R}^{n}\right)\right\}
$$

We equip $\left\{\mathrm{dh} \mid \mathrm{h} \in \mathrm{C}^{\infty}\left(\mathrm{M}, \mathbb{R}^{\mathrm{n}}\right)\right\}$ with the uniquely determined topology making

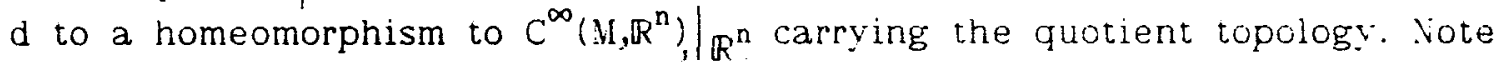
that both topological spaces are Frechet manifolds.

Next we identify them via d. Hence we have identified also the two Frechet manifolds $\left.E\left(M, \mathbb{R}^{n}\right)\right|_{\mathbb{R}^{n}}$ and $\left\{d j \mid j \in E\left(M, \mathbb{R}^{n}\right)\right\}$ vielding

$$
\left.T E\left(M, \mathbb{R}^{n}\right)\right|_{\mathbb{R}^{n}}=\left\{d j \mid j \in E\left(M, \mathbb{R}^{n}\right)\right\} \times\left\{d h \mid h \in C^{\infty}\left(M, \mathbb{R}^{n}\right)\right\} .
$$

Therefore we obtain the following 
Lemma 5.1 :

Given a smooth map

$$
F_{\mathbb{R}^{n}}:\left.\left.\left.C^{\infty}\left(M, \mathbb{R}^{n}\right)\right|_{\mathbb{R}^{n} \times E\left(M, \mathbb{R}^{n}\right)}\right|_{\mathbb{R}^{n} \times C^{\infty}\left(M, \mathbb{R}^{n}\right)}\right|_{\mathbb{R}^{n}} \longrightarrow \mathbb{R},
$$

linear in the third argument, then the resulting (parameter depending) one form $\mathrm{F}$ given by

$$
F=F_{\mathbb{R}^{\mathrm{n}} \circ(\mathrm{d}, \mathrm{Td})}
$$

is a (parameter depending) $\mathbb{R}^{n}$-invariant one form on $E\left(M, \mathbb{R}^{n}\right)$.

Here $T d$ is the tangent map of

$$
d:\left.E\left(M, \mathbb{R}^{n}\right) \longrightarrow E\left(M, \mathbb{R}^{n}\right)\right|_{\mathbb{R}^{n}}
$$

Remark 5.1 :

In the following we write

$$
d^{*} F_{\mathbb{R}^{n}} \quad \text { instead of } F_{\mathbb{R}^{n} \circ(d, T d)} \text {. }
$$

Remark 5.2 :

The above lemma allows us to study the constitutive laws of the type $d^{*} F_{\mathbb{R}^{n}}$ rather than constitutive laws invariant under $\operatorname{Tr}$. 
Let throughout this section if $E\left(M, \mathbb{R}^{\mathrm{n}}\right)$ be a fixed smooth embedding and $\in A^{1}\left(M, \mathbb{R}^{n}\right)$ be a fixed smooth $\mathbb{R}^{n}$-valued one-form. We follow [Bi,2].

As the first observation we formulate the following

Proposition 6.1 :

Let $\alpha \in A^{1}\left(M, \mathbb{R}^{n}\right)$ and $i \in E\left(M, \mathbb{R}^{n}\right)$ be given.

Then the following decomposition holds

$$
\alpha=d h+\beta
$$

where $h \in C^{\infty}\left(M, \mathbb{R}^{n}\right)$, the so-called integrable part of $\alpha$, is uniquely determined up to a constant. Moreover this decomposition is maximal in the sense that the integrable part of $\beta$ is a constant.

\section{Proof :}

Let $e_{1}, \ldots, e_{n}$ be an orthonormal basis of $\mathbb{R}^{\mathrm{n}}$. Then we get

$$
\alpha(X)=\sum_{s=1}^{n} \alpha^{s}(X) e_{5}, \quad \forall X \in \Gamma T M,
$$

for an uniquely determined family $\alpha^{1}, \ldots, \alpha^{\mathrm{n}}$ of smooth $\mathbb{R}$ - valued one-forms on $M$, i.e. $\alpha^{s} \in A^{1}(M, \mathbb{R}), s=1, \ldots, n$. Clearly

$$
\alpha^{s}(X)=\left\langle\alpha(X), \mathrm{e}_{\mathrm{s}}\right\rangle, \quad \forall \not \subset \in \Gamma T \mathrm{M}, \mathrm{s}=1, \ldots, \mathrm{n} .
$$

In addition $\alpha^{s}, s=1, \ldots, n$, can be represented as

$$
\alpha^{s}(X)=m(i)\left(Y_{s}, X\right), \quad \forall X \in \Gamma T M
$$

for a well defined $y_{s} \in \Gamma T M$. This vector field splits according to Hodge's decomposition uniquely into

$$
\left\{\begin{array}{l}
Y_{s}=\operatorname{grad}_{i} \tau_{s}+Y_{s}^{0}, \\
\operatorname{div} v_{i} Y_{s}^{0}=0,
\end{array}\right.
$$

where $\tau_{s} \in C^{\infty}\left(M, \mathbb{R}^{n}\right)$ and $Y_{s}^{0} \in \Gamma T M$.

Hence

$$
\alpha^{s}(X)=d \tau_{s}(X)+m(i)\left(Y_{s}^{0}, X\right), \quad \forall X \in \Gamma T M .
$$

Next we define the integrable part $h$ of $\alpha$ by

$$
h:=\sum_{s=1}^{n} \tau_{s} e_{s},
$$

and the non-integrable part $\beta$ by 
(6.8)

$$
\beta(X):=\sum_{s=1} m(i)\left(Y_{s}^{0}, X\right) e_{s}, \quad \forall X \in \Gamma T M
$$

Inserting (6.6) into (6.2) and using (6.7) and (6.8) yields the decomposition (6.1). It remains only to show that (6.1) does not depend on the choice of the basis of $\mathbb{R}^{n}$. To this end let $\bar{e}_{1}, \ldots, \bar{e}_{n} \in \mathbb{R}^{n}$ be another orthonormal basis of $\mathbb{R}^{n}$ and define $\bar{\alpha}, \bar{\tau}, \bar{Y}^{0}, \bar{h}$ and $\bar{\beta}$ accordingly.

Then

$$
\begin{aligned}
\bar{\alpha}(X) & =\langle\alpha(X), \bar{e}\rangle \\
& =\langle d h(X), \bar{e}\rangle+\langle\beta(X), \bar{e}\rangle \\
& =\left\langle\sum_{s=1}^{n} d \tau_{s}(X) e_{s}, \bar{e}\right\rangle+\left\langle\sum_{s=1}^{n} m(i)\left(Y_{s}^{0}, X\right) e_{s}, \bar{e}\right\rangle \\
& =m(i)\left\langle\sum_{s=1}^{n} \operatorname{grad}_{i} \tau_{s}\left\langle e_{s}, \bar{e}\right\rangle, X\right) \\
& +m(i)\left\langle\sum_{s=1}^{n} y_{s}^{0}\left\langle e_{s}, \bar{e}\right\rangle, X\right) \\
& =\langle d \bar{h}(X), \bar{e}\rangle+\langle\bar{\beta}(X), \bar{e}\rangle \\
& =m(i)\left(\operatorname{grad}_{i} \bar{\tau}, X\right)+m(i)\left(\bar{Y}^{0}, X\right) .
\end{aligned}
$$

Since on one hand

$$
\sum_{s=1}^{n} \operatorname{grad}_{i} \tau_{s}\left\langle e_{s}, \bar{e}\right\rangle=\operatorname{grad}_{i}\left(\sum_{s=1}^{n} \tau_{s}\left\langle e_{s}, \bar{e}\right\rangle\right),
$$

on the other hand

$$
\operatorname{div}_{i}\left(\sum_{s=1}^{n} Y_{s}^{0}\left\langle e_{s}, \bar{e}\right\rangle\right)=\sum_{s=1}^{n}\left(\operatorname{div}_{i} Y_{s}^{0}\right)\left\langle e_{s}, \bar{e}\right\rangle=0,
$$

we conclude due to the uniqueness of Hodge's decomposition the following relations

$$
\left\{\begin{array}{l}
\sum_{s=1}^{n} \operatorname{grad}_{i}\left(\tau_{s}\left\langle e_{s}, \bar{e}\right\rangle\right)=\operatorname{grad}_{i} \bar{\tau}, \\
\sum_{s=1}^{n} Y_{s}^{0}\left\langle e_{s}, \bar{e}\right\rangle=\bar{Y}^{0} \ldots
\end{array}\right.
$$

Consequently the uniqueness of the decomposition (6.1) follows, namely

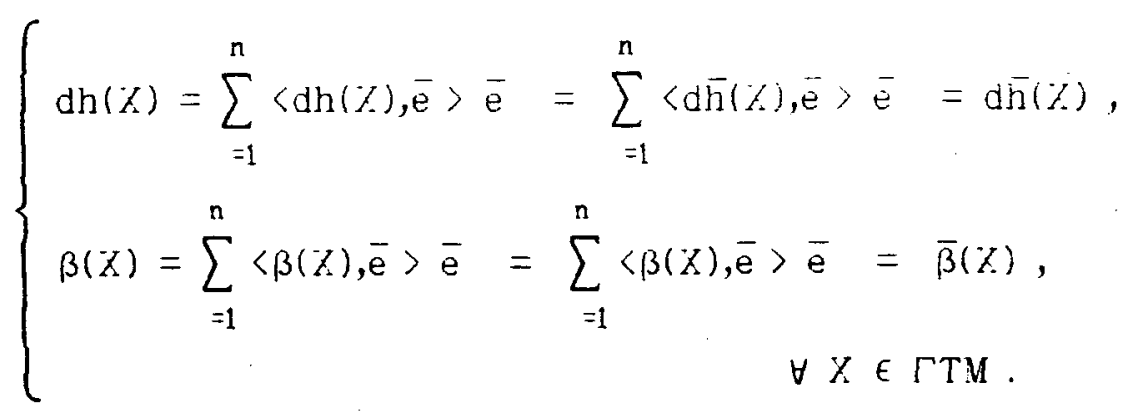


Let us detail now the decomposition (6.1).

For this purpose we note first that $h$ can be given the form

$$
\mathrm{h}=\mathrm{di} \mathrm{X}_{\mathrm{h}}+\mathrm{h}^{\perp} \text {, }
$$

where $x_{h} \in \Gamma T M$ is well defined and $h^{\perp}$ denotes the pointwise formed component of $\mathrm{h}$ normal to $\mathrm{i}(\mathrm{M})$.

Using the fact that $X_{h}$ splits into

$$
\left\{\begin{array}{l}
x_{h}=X_{h}^{0}+\operatorname{grad}_{i} \psi_{h}, \\
\operatorname{div}_{\mathrm{i}} X_{\mathrm{h}}^{0}=0,
\end{array}\right.
$$

where $\psi_{h} \in C^{\infty}(M, R), \chi_{h}^{0} \in \Gamma T M$, we deduce from $(6.14)$ that

$$
\begin{aligned}
\operatorname{dh}(X)= & \operatorname{di} \nabla(\mathrm{i})_{X} X_{\mathrm{h}}^{0}+\mathrm{di}\left(\nabla(\mathrm{i})_{X} \operatorname{grad}_{\mathrm{i}} \psi_{\mathrm{h}}+\mathrm{w}_{\mathrm{h}}(\mathrm{i}) X\right) \\
& +\mathrm{S}(\mathrm{i})\left(X_{\mathrm{h}}^{0}, X\right)+\left(\mathrm{dh}^{\perp}(X)\right)^{\perp} .
\end{aligned}
$$

For the sake of readability we remind that $W_{h}(i)$ defined via

$$
\text { di } W_{h}(i) X:=\left(d h^{\perp}(X)\right)^{T} \text {, }
$$

is a smooth strong bundle endomorphism of TM, which is selfadjoint with respect to $\mathrm{m}(\mathrm{i})$.

Let us show next that the divergence-free part $X_{\mathrm{h}}^{0}$ of $X_{\mathrm{h}}$ is uniquely determined by $h$. To this end we use the fact that according to the above proposition the integrable part $h$ of $\alpha$ is uniquely determined up to a constant, i.e.

$$
h^{\prime}=h+z,
$$

for some $z \in \mathbb{R}^{\mathrm{n}}$. Regarding $z$ as a constant map in $\mathcal{C}^{\infty}\left(\mathrm{W}, \mathbb{R}^{\mathrm{n}}\right)$ we write it in the form

$$
z=d i x_{z}+z^{\perp} \text {. }
$$

But the vector field $Z$ on $\mathbb{R}^{\mathrm{n}}$ assigning to any $Z \in \mathbb{R}^{\mathrm{n}}$ the vector $Z(z)=Z \in \mathbb{R}^{\mathrm{n}}$ is the gradient of some map $\rho \in C^{\infty}\left(\mathrm{M}, \mathbb{R}^{\mathrm{n}}\right)$ and hence

$$
X_{z}=\operatorname{grad}_{i}(P \circ i) .
$$

Therefore

$$
\begin{aligned}
h^{\prime} & =\operatorname{di} X_{\mathrm{h}}^{0}+\mathrm{di} \operatorname{grad}_{\mathrm{i}}\left(\psi_{\mathrm{h}}+\varphi \circ \mathrm{i}\right)+\mathrm{h}^{\perp}+z^{\perp} \\
& =\operatorname{di} X_{\mathrm{h}^{\prime}}^{0}+\operatorname{di}_{\operatorname{grad}_{\mathrm{i}} \psi_{\mathrm{h}^{\prime}}+\mathrm{h}^{\prime}}
\end{aligned}
$$

or, equivalently 


$$
\begin{aligned}
& \operatorname{di}\left(X_{h^{\prime}}^{0}-X_{h}^{0}\right)+\operatorname{di} \operatorname{grad}_{i}\left(\psi_{h^{\prime}}-\psi_{h}-\rho \circ i\right) \\
& =h^{\perp}+z^{\perp}-h^{\perp}=0 .
\end{aligned}
$$

But (6.22) implies

$$
x_{h}^{0},-X_{h}^{0}+\operatorname{grad}_{i}\left(\psi_{h},-\psi_{h}-\varphi_{0} i\right)=0 .
$$

Lising once more the uniqueness of Hodge's decomposition we conclude then $(\mathrm{cf},[\mathrm{Bi}, 2])$

\section{Proposition 6.2 :}

Let $\alpha \in A^{1}\left(M, \mathbb{R}^{n}\right)$ and $i \in E\left(\mathbb{M}, \mathbb{R}^{n}\right)$ be given, and denote by $h \in C^{\infty}\left(M, \mathbb{R}^{n}\right)$ the integral part of $\alpha$, which is uniquely detemined up to a constant

$$
\alpha=\mathrm{dh}+\beta \text {. }
$$

Splitting $h$ into

$$
\mathrm{h}=\mathrm{di} z_{\mathrm{h}}+\mathrm{h}^{\perp}
$$

where $X_{\mathrm{h}} \in \Gamma \mathrm{TM}$ is well defined, then the divergence-free part $X_{\mathrm{h}}^{0}$ of $X_{\mathrm{h}}$ is uniquely determined.

Next we characterize $\propto \in A^{1}\left(M, \mathbb{R}^{\mathrm{n}}\right)$ relative to if $E\left(M, \mathbb{R}^{\mathrm{n}}\right)$ from a quite different point of view. To this end let us introduce the following two tensor $T_{\alpha}$ on $M$

$$
T_{\alpha}(X, Y):=\langle\alpha(X) \text {, di } Y\rangle,
$$$$
\forall X, Y \in \Gamma T M .
$$

Clearly $T_{\alpha}$ is smooth. Next we denote by

$$
\mathrm{P}: \mathrm{TM} \longrightarrow \mathrm{TM}
$$

the unique smooth strong bundle endomorphism for which

$$
T_{\alpha}(X, Y)=m(i)(P X, Y)
$$

and by $\tilde{\mathrm{P}}$ the fibre-wise formed adjoint of $\mathrm{P}$ with respect to $\mathrm{m}(\mathrm{i})$. The symmetric and the antisymmetric part of $T_{\alpha}$, the tensors $T_{\alpha}^{S}$ and $T_{\alpha}^{a}$ respectively have the form

$$
\begin{aligned}
& T_{\alpha}^{S}(X, Y)=m(i)\left(\frac{1}{2}(P+\tilde{P}) X, Y\right), \\
& T_{\alpha}^{a}(X, Y)=m(i)\left(\frac{1}{2}(P-\tilde{P}) X, Y\right) .
\end{aligned}
$$

Setting now

$$
\left\{\begin{array}{l}
B_{\alpha}:=\frac{1}{2}(P+\tilde{P}) P \\
C_{\alpha}:=\frac{1}{2}(P-\tilde{P}) P,
\end{array}\right.
$$


we obtain that

$$
\alpha(X)=\alpha^{\prime}(X)+\mathrm{di}_{\alpha} X+\mathrm{di} \mathrm{B}_{\alpha} X, \quad \forall X \in \Gamma \mathrm{T} \mathrm{X} .
$$

Clearly $\alpha^{\prime}(X)(p)$ is a vector in the normal space of TiT $\mathrm{p}^{M}, \forall p \in M$.

Hence there is a unique smooth map

$$
c_{\alpha} \in C^{\infty}(M, s o(n))
$$

where so(n) denotes the Lie algebra of the group of all proper rotations of $\operatorname{SO}(n)$, such that

$$
\alpha^{\prime}(X)=c_{\alpha} \operatorname{di} X,
$$

with

$$
c_{\alpha}(N(j)) \perp \operatorname{ker} c_{\alpha},
$$$$
\forall X \in \Gamma T M .
$$

We may now state the following

\section{Proposition 6.3:}

Let $\alpha \in A^{1}\left(M, \mathbb{R}^{n}\right)$ and $i \in E\left(M, \mathbb{R}^{n}\right)$ be given. Then there exist two uniquely determined smooth, strong bundle endomorphisms

and

$$
\mathrm{C}_{\alpha}: \mathrm{TM} \longrightarrow \mathrm{TM}
$$

$$
\mathrm{B}_{\alpha}: \mathrm{TM} \longrightarrow \mathrm{TM}
$$

which are skew- and respectively selfadjoint with respect to $m(i)$, and a uniquely determined map $c_{\alpha} \in C^{\infty}(\mathrm{H}, \mathrm{so}(n))$, such that the following relation holds

$$
\alpha(\not)=\mathrm{c}_{\alpha} \text { di } \not X+\mathrm{di}_{\alpha} \not X+\mathrm{di} \mathrm{B}_{\alpha} \not Z, \quad \forall \not Z \in \Gamma T W .
$$

\section{Remark 6.1:}

Given $\alpha \in A^{1}\left(M, \mathbb{R}^{\mathrm{n}}\right)$ and $i \in E\left(M, \mathbb{R}^{\mathrm{n}}\right)$, then

$$
\delta T_{\alpha}^{a}=0 \quad \text { iff } \dot{\delta} \alpha=0
$$

Indeed, let us consider the one form $\langle i, \alpha\rangle \in A^{1}\left(M, R^{n}\right)$, which assigns to any $X \in \Gamma T M$ the real function $\langle i, \alpha(X)\rangle$.

Since

$$
\delta\langle i, \alpha\rangle=T_{\alpha}^{a} \quad \text { iff } \delta \alpha=0
$$

(6.35) then follows immediately. 
Next we link the two characterizations of $\mathbb{R}^{\mathrm{n}}$-valued one-forms relative to embeddings, as expressed by the two propositions above. To this end let $\alpha \in A^{l}\left(M, \mathbb{R}^{n}\right)$ and $i \in E\left(M, \mathbb{R}^{n}\right)$ be given.

Using (6.1) and (6.14) $\propto(X)$ turns into

$$
\begin{aligned}
\alpha(X)= & \operatorname{di} \nabla(\mathrm{i})_{X_{\mathrm{h}}}+\mathrm{di}_{\mathrm{h}}(\mathrm{i}) X+\mathrm{S}(\mathrm{i})\left(X_{\mathrm{h}}, X\right) \\
& +\left(\mathrm{dh}^{\perp}(X)\right)^{\perp}+\beta(X) .
\end{aligned}
$$

Inserting $(6.37)$ in $(6.24)$ we get

$$
\begin{aligned}
& \mathrm{T}_{\alpha}(\mathrm{X}, \mathrm{Y})=\langle\alpha(X) \text {,di } \mathrm{Y}\rangle \\
& =\left\langle\operatorname{di} \nabla(\mathrm{i})_{X} X_{h}+W_{h}(i) X, d i Y\right\rangle+T_{\beta}(X, Y) \\
& =m(\mathrm{i})\left(\nabla(\mathrm{i})_{X} X_{\mathrm{h}}, Y\right)+m(\mathrm{i})\left(\mathrm{W}_{\mathrm{h}}(\mathrm{i}) X, Y\right)+\mathrm{T}_{\beta}(X, Y) \text {, } \\
& \forall X, Y \in \Gamma T \mathrm{~T} \text {. }
\end{aligned}
$$

Therefore

$$
\begin{aligned}
T_{\alpha}^{S}(X, Y)= & \frac{1}{2}\left[m(i)\left(\nabla(i)_{X} X_{h}, Y\right)+m(i)\left(\nabla(i)_{y} X_{h}, X\right)\right] \\
& +m(i)\left(W_{h}(i) X, Y\right)+T_{\beta}^{S}(X, Y) \\
= & \frac{1}{2} L_{X_{h}}(m(i))(X, Y)+m(i)\left(W_{h}(i) X, Y\right)+T_{\beta}^{S}(X, Y), \\
T_{\alpha}^{a}(X, Y)= & \frac{1}{2}\left[m(i)\left(\nabla(i)_{X} X_{h}, Y\right)-m(i)\left(\nabla(i)_{Y} X_{h}, X\right)\right]+T_{\beta}^{a}(X, Y),
\end{aligned}
$$

Rewriting the Lie derivative $L_{z_{h}}(m(i))$ of $m(i)$ in the direction of $z_{h}$ with the help of the Theorem of Fischer and Riesz as

$$
\mathrm{L}_{\mathrm{h}}\left(\mathrm{m}(\mathrm{i})(X, \mathrm{Y})=\mathrm{m}(\mathrm{i})\left(\mathrm{L}_{\mathrm{h}} X, \mathrm{Y}\right) \quad \forall \mathrm{Z}_{\mathrm{h}} \in \Gamma \mathrm{TM},\right.
$$

by a uniquely determined strong smooth bundle endomorphism

$$
\mathrm{L}_{\mathrm{h}_{\mathrm{h}}}: \mathrm{TM} \longrightarrow \mathrm{TM}
$$

from $(6.34)$ and $(6.37)$ we infer the following formulas for $c_{Q x}, C_{\alpha}$ and $B_{\alpha}$ :

$$
c_{\alpha} \text { di } X=\left(\mathrm{dh}^{\perp}(X)\right)^{\perp}+\mathrm{s}(\mathrm{i})\left(X_{\mathrm{h}}, X\right)+c_{\beta} \mathrm{di} X,
$$

$$
c_{\alpha} Y=\frac{1}{2}\left[\nabla(\mathrm{i}) X_{\mathrm{h}}-\tilde{\nabla}(\mathrm{i}) X_{\mathrm{h}}\right] Y+c_{\beta} Y,
$$

(6.45)

$$
\begin{aligned}
\mathrm{B}_{\alpha} X & =\frac{1}{2}\left[\nabla(\mathrm{i}) X_{\mathrm{h}}+\tilde{\nabla}(\mathrm{i}) \mathrm{X}_{\mathrm{h}}\right] X+\mathrm{w}_{\mathrm{h}}(\mathrm{i}) Z+\mathrm{B}_{\beta} Z \\
& =\left(\frac{1}{2} \mathrm{~L}_{\mathrm{X}_{\mathrm{h}}}+\mathrm{w}_{\mathrm{h}}(\mathrm{i})+\mathrm{B}_{\beta}\right) X .
\end{aligned}
$$

Instead of $\nabla(i) Z_{\mathrm{v}}$ we often write $\nabla(\mathrm{i}) K_{\mathrm{h}}(v)$ for any $v \in T_{\mathrm{p}}$ M. Similarily we use $\tilde{\nabla}(i) Y_{h}(v)$ instead of $\tilde{\nabla}(i){ }_{v} K_{h}$.

Using next the Hodge decomposition of $Z_{h}$, i.e. (6.15) and taking into account that

$$
m(i)\left(\left(\nabla(i) \operatorname{grad}_{i} \psi_{h}-\tilde{\nabla}(i) \operatorname{grad}_{i} \psi_{h}\right) X, Y\right)=0 \text {, }
$$

we obtain finally the following 
Let $\alpha \in A^{1}\left(M, \mathbb{R}^{n}\right)$ and $i \in E\left(M, \mathbb{R}^{n}\right)$ be given. Then the following relations hold

$$
\begin{aligned}
& \alpha=\mathrm{dh}+\beta, \\
& \alpha(X)=c_{\alpha} \text { di } X+\operatorname{di~} C_{\alpha} X+\operatorname{di~} B_{\alpha} X, \quad \forall X \in \Gamma T M,
\end{aligned}
$$

where the integrable part $h \in C^{\infty}\left(M, \mathbb{R}^{n}\right)$ is uniquely determined up to a constant , $C_{\alpha} \in C^{\infty}(M, s o(n))$ is a uniquely determined, $C_{\alpha}: T M \longrightarrow T M$ is a uniquely determined smooth, strong and skew-adjoint bundle endomorphism, $\mathrm{B}_{\alpha}: \mathrm{TM} \rightarrow \mathrm{TM}$ is a uniquely determined smooth, strong and selfadjoint bundle endomorphism.

Writing

$$
h=d i X_{h}+h^{\perp}
$$

where $X_{h} \in \Gamma T M$, and using Hodge's decomposition

$$
\left\{\begin{array}{l}
X_{\mathrm{h}}=X_{\mathrm{h}}^{0}+\operatorname{grad}_{\mathrm{i}} w_{\mathrm{h}}, \\
\operatorname{div}_{\mathrm{i}} X_{\mathrm{h}}^{0}=0
\end{array}\right.
$$

we obtain finally

$$
c_{\alpha} d i=\left(d h^{\perp}\right)^{\perp}+s(i)\left(X_{h}, .\right)+c_{\beta} d i,
$$

$$
c_{\alpha}=\frac{1}{2}\left[\nabla(i) x_{h}^{0}-\tilde{\nabla}(i) x_{h}^{0}\right]+c_{\beta},
$$

$$
B_{\alpha}=\frac{1}{2} L_{X_{h}^{0}}+\operatorname{grad}_{i} \psi_{h}+w_{h}(i)+B_{\beta} \text {. }
$$

Hence

$$
\begin{aligned}
\operatorname{tr} \mathrm{B}_{\alpha} & =\operatorname{div} \gamma_{h}+\operatorname{tr} w_{h}(\mathrm{i})+\operatorname{tr} B_{\beta} \\
& =-\Delta(\mathrm{i}) \omega_{h}+\operatorname{tr} w_{h}(i)+\operatorname{tr} B_{\beta},
\end{aligned}
$$

where $\Delta(i)$ is the Laplace-Beltrami operator of $m(i)$.

Let us calculate now the covariant divergence of $B_{\alpha}$ and $C_{\alpha}$. To this end we recall at first the covariant divergence div ${ }_{i}$ A of a smooth strong bundle endomorphism

$$
\mathrm{A}: \mathrm{TM} \longrightarrow \mathrm{TM}
$$

Let $e_{1}, \ldots, e_{m}$ be a moving orthonormal frame of $T M$, and set

$$
\operatorname{div}_{i} A=\sum_{r=1}^{n} \nabla(i) e_{p}(A) e_{Y} .
$$

At first we compute div $\nabla_{i}(i) X_{h}$. Using the equation 
(6.54)

$$
\begin{aligned}
& m(i)\left(\nabla(i) e_{Y}\left(\nabla(i) X_{h}\right) e_{y}, Y\right) \\
& =m(i)\left(\nabla(i) e_{Y}\left(\nabla(i) e_{r} X_{h}\right)-\nabla(i) \nabla(i) e_{r} e_{r} X_{h}, Y\right), \\
& \forall Y \in \Gamma T M,
\end{aligned}
$$

we get

(6.55)

$$
\operatorname{div}_{i}\left(\nabla(i) X_{h}\right)=\Delta(i) X_{h}
$$

where $\Delta(i) X_{h}$ is the Laplace-Beltrami operator of $\mathrm{m}(\mathrm{i})$ applied to $X_{\mathrm{h}}$ which by definition is $-\operatorname{tr} \nabla(i)_{n}^{2} x_{h}$.

In order to compute $\operatorname{div}_{\dot{i}} \nabla(i) X_{h}$ we consider the equations

$$
\begin{aligned}
m(i) & \left(\nabla(i) e_{Y}\left(\tilde{\nabla}(i) X_{h}\right)\left(e_{Y}\right), Y\right) \\
= & e_{r}\left(m(i)\left(\tilde{\nabla}(i) X_{h}\left(e_{Y}\right), Y\right)-m(i)\left(\tilde{\nabla}(i) X_{h}\left(\nabla(i) e_{Y} e_{r}\right), Y\right)\right. \\
& -m(i)\left(\tilde{\nabla}(i) X_{h}\left(e_{Y}\right), \nabla(i) e_{Y}^{Y}\right) \\
= & m(i)\left(e_{Y}, \nabla(i) e_{Y} \nabla(i)_{Y} X_{h}\right)-m(i)\left(e_{Y}, \nabla(i) \nabla(i) e_{Y} Y X_{h}\right) \\
= & m(i)\left(e_{Y}, \nabla(i) e_{e_{Y}}\left(\nabla(i) X_{h}\right) Y\right),
\end{aligned}
$$

$(6.57)$

$$
\begin{aligned}
m(i) & \left(\nabla(i)_{Y}\left(\tilde{\nabla}(i) X_{h}\right)\left(e_{Y}\right), e_{Y}\right) \\
= & m(i)\left(e_{Y}, \nabla(i)_{Y} \nabla(i) e_{Y} X_{h}\right)-m(i)\left(e_{Y}, \nabla(i) \nabla(i) e_{Y} e_{Y} X_{h}\right) \\
= & m(i)\left(e_{Y}, \nabla(i)_{Y}\left(\nabla(i) X_{h}\right)\left(e_{Y}\right)\right)
\end{aligned}
$$

and find

$(6.58)$

$$
\begin{aligned}
& \sum_{r=1}^{n}\left[m(i)\left(\nabla(i) e_{Y}\left(\tilde{\nabla}(i) X_{h}\right)\left(e_{r}\right), Y\right)-m(i)\left(\nabla(i) Y\left(\tilde{\nabla}(i) X_{h}\right)\left(e_{r}\right), e_{r}\right)\right] \\
& \quad=\operatorname{Ric}(m(i))\left(Y, X_{h}\right),
\end{aligned}
$$

where Ric(m(i)) denotes the Ricci tensor of $m(i)$. Hence

(6.59)

$$
m(i)\left(\operatorname{div}_{i}\left(\tilde{\nabla}(i) X_{h}\right), Y\right)=\operatorname{tr} \nabla(i){ }_{Y}\left(\nabla(i) X_{h}\right)+\operatorname{Ric}(m(i))\left(X_{-}, X_{h}\right)
$$

But (6.59) yields

$(6.60)$

$$
\operatorname{div}_{\mathrm{i}}\left(\tilde{\nabla}(\mathrm{i}) X_{\mathrm{h}}\right)=\operatorname{grad}_{\mathrm{i}} \mathrm{div}{ }_{\mathrm{i}} \chi_{\mathrm{h}}+\mathrm{R}(\mathrm{i}) X_{\mathrm{h}},
$$

where $R(i) x_{h}$ is defined via

(6.61)

$$
m(i)\left(R(i) X_{h}, Y\right)=\operatorname{Ric}(m(i))\left(X_{h}, Y\right),
$$

$\forall Y \in \Gamma T M$.

From $(6.49),(6.50),(6.55)$ and $(6.60)$ we deduce

$$
\operatorname{div}_{i} L_{X_{h}}=\Delta(i) X_{h}+R(i) X_{h}+\operatorname{grad}_{i} \operatorname{div}_{i} X_{h} \text {, }
$$

(6.63)

$$
2 \operatorname{div}_{i} C_{h}=\Delta(i) X_{h}-R(i) X_{h}-\operatorname{grad}_{i} \operatorname{div}_{i} X_{h}
$$


and consequently

$$
\begin{aligned}
& \operatorname{div}_{i}\left(\frac{1}{2} L_{X_{h}}+C_{h}\right)=\Delta(i) X_{h}, \\
& \operatorname{div}_{i}\left(\frac{1}{2} L_{X_{h}}-C_{h}\right)=R(i) X_{h}+\operatorname{grad}_{i} \operatorname{div}_{i} X_{h} .
\end{aligned}
$$

Let us restrict our attention to the case where $M$ has codimension 1 . Since $M$ is oriented we have an oriented unit normal vector field $\mathrm{N}(\mathrm{i})$ along $\mathrm{i}$.

Hence $h \in C^{\infty}\left(M, \mathbb{R}^{n}\right)$ splits uniquely into

(6.66)

$$
h=d i X_{h}+\Theta_{h} N(i)
$$

where $X_{\mathrm{h}} \in \Gamma \mathrm{TM}, \Theta_{\mathrm{h}} \in \mathrm{C}^{\infty}(\mathrm{M}, \mathbb{R})$.

Thus

$(6.67)$

$$
W_{h}(i)=W(i) \quad \text { if } \Theta_{h}=1 \text {. }
$$

Defining the mean curvature $\mathrm{H}(\mathrm{i})$ of $\mathrm{i}$ by

$$
\operatorname{tr} W(i)=H(i)
$$

we immediately find

$$
\begin{aligned}
& m(i)\left(\operatorname{div}_{i}\left(\Theta_{h} W(i)\right), Y\right) \\
& \quad=\sum_{Y=1}^{n-1} m(i)\left(\nabla(i) e_{Y}\left(\Theta_{h} W(i)\right) e_{Y}, Y\right) \\
& =m(i)\left(\operatorname{grad}_{i} \Theta_{h}, W(i) Y\right)+m(i)\left(\Theta_{h} \operatorname{div}_{i} W(i), Y\right)
\end{aligned}
$$

and hence

$$
\operatorname{div}_{i}\left(\Theta_{h} W(i)\right)=W(i) \operatorname{grad}_{i} \Theta_{h}+\Theta_{h} \operatorname{div}_{i} W(i) .
$$

On the other hand by Codazzi's equation (cf. $[\mathrm{Kl}]$ )

$$
\begin{aligned}
\sum_{r=1}^{m} m(i)\left(\nabla(i) e_{e_{r}}(W(i)) e_{r}, Y\right) & =\sum_{r=1}^{n-1} m(i)\left(\nabla(i)_{Y}(W(i)) e_{r}, e_{r}\right) \\
& =m(i)\left(\operatorname{grad}_{i} H(i), Y\right)
\end{aligned}
$$

and consequently

$$
\operatorname{div}_{i}\left(\Theta_{h} W(i)\right)=W(i) \operatorname{grad}_{i} \Theta_{h}+\Theta_{h} \operatorname{grad}_{i} H(i)
$$

From (6.49), (6.60), (6.67), (6.68) and (6.72) we infer then

$$
\operatorname{div}_{i}\left(B_{h}+C_{h}\right)=\Delta(i) Z_{h}+W(i) \operatorname{grad}_{i} \Theta_{h}+\Theta_{h} \operatorname{grad}_{i} H(i)
$$

$(6.74)$

$$
\begin{aligned}
\operatorname{div}_{i}\left(B_{h}-C_{h}\right)= & R(i) X_{h}+\operatorname{grad}_{i} \operatorname{div}_{i} X_{h} \\
& +W(i) \operatorname{grad}_{i} \Theta_{h}+\Theta_{h} \operatorname{grad}_{i} H(i),
\end{aligned}
$$


(6.75)

$$
\begin{aligned}
\operatorname{div}_{i} B_{h}= & \frac{1}{2} \Delta(i) X_{h}+\frac{1}{2} R(i) X_{h} \\
& +W(i) \operatorname{grad}_{i} \Theta_{h}+\Theta_{h} \operatorname{grad}_{i} H(i)
\end{aligned}
$$

We conclude this section by proving the following

\section{Lemma 6.5 :}

Let $\alpha \in A^{1}\left(M, \mathbb{R}^{n}\right)$ and $i \in E\left(M, \mathbb{R}^{n}\right)$ be given.

If $\alpha$ has no integrable part, i.e. $\alpha=\beta$, then

(6.76)

$$
\operatorname{div}_{i}\left(C_{\beta}+B_{\beta}\right)=0
$$

\section{Proof :}

Denoting by $\bar{e}_{1}, \ldots, \bar{e}_{n}$ an orthonormal basis of $\mathbb{R}^{n}$ we have, in accordance with (6.8),

$(6.7 \bar{i})$

$$
\begin{aligned}
\mathrm{T}_{\beta}(X, Y) & =\langle\beta(X), \mathrm{di} Y\rangle \\
& =\mathrm{m}(\mathrm{i})\left(\left(\mathrm{B}_{\beta}+\mathrm{C}_{\beta}\right) X, Y\right) \\
& =\sum_{s=1}^{\mathrm{n}} \mathrm{m}(\mathrm{i})\left(\mathrm{Y}_{\mathrm{s}}^{0}, X\right)\left\langle\bar{e}_{\mathrm{s}}, \mathrm{di} \mathrm{Y}\right\rangle \\
& =\mathrm{m}(\mathrm{i})\left(\sum_{\mathrm{s}=1}^{\mathrm{n}}\left\langle\overline{\mathrm{e}}_{\mathrm{s}}, \mathrm{di} Y\right\rangle Y_{s}^{0}, X\right), \quad \forall X, Y \in \Gamma T M .
\end{aligned}
$$

If now $e_{1}, \ldots, e_{m}$ is a moving orthonormal frame in $T M$, then we get

$(6.78)$

$$
\begin{gathered}
m(i)\left(\operatorname{div}_{i}\left(B_{\beta}+C_{\beta}\right), Y\right)=\sum_{r=1}^{m} m(i)\left(\nabla(i) e_{r}\left(B_{\beta}+C_{\beta}\right) e_{r}, Y\right) \\
=\sum_{r=1}^{m} \sum_{s=1}^{n} m(i)\left(\nabla(i) e_{r}\left\langle\bar{e}_{s}, d i \quad Y\right\rangle Y_{s}^{0}, e_{Y}\right)
\end{gathered}
$$

$\mathrm{BV}$ interchanging the summation the assertion then follows. 
Let $M$ be a moving deformable medium of codimension 1, i.e. $\operatorname{dim} M=n-1$, and assume that its motion is due only to an internal constitutive law $F$. As we have already seen $F$ is $\mathbb{R}^{\mathrm{n}}$-invariant and admits the representation

where

$$
F=d^{*} F_{\mathbb{R}^{n}}
$$

$$
F_{\mathbb{R}^{n}}:\left.\left.C^{\infty}\left(M, \mathbb{R}^{n}\right)\right|_{\mathbb{R}^{n} \times E\left(M, \mathbb{R}^{n}\right)}\right|_{\mathbb{R}^{n} \times C^{\infty}\left(M, \mathbb{R}^{n}\right) \longrightarrow \mathbb{R}}
$$

is a smooth map. Next we assume that $F_{\mathbb{R}^{n}}$ has an integral representation

$$
F_{\mathbb{R}^{n}(d k)(d j, d l):=} \int \alpha(d j, d k) \cdot d l \mu(j), \quad \forall j \in E\left(M_{k}, \mathbb{R}^{n}\right),
$$

where $\alpha$ is an $\mathbb{R}^{\mathrm{n}}$-valued one form, the so-called stress-form, i.e.

$$
\alpha:\left.T E\left(M, \mathbb{R}^{n}\right)\right|_{\mathbb{R}^{n}} \longrightarrow A^{1}\left(M, \mathbb{R}^{n}\right)
$$

We note that the integrand

$$
\alpha(d j, d k) \cdot d l
$$

in (7.1) is defined in the following way :

Let first represent $\alpha(\mathrm{dj}, \mathrm{dk})$ and $\mathrm{dl}$ according to Proposition 6.3 as

and

$$
\alpha(d j, d k)=c_{\alpha}(d j, d k) \cdot d l+d j \cdot C_{\alpha}(d j, d k)+d j \cdot B_{\alpha}(d j, d k)
$$

$$
d l=c_{l} \cdot d j+d j \cdot C_{1}+d j \cdot B_{1}
$$

respectively. Then we set

$$
\begin{aligned}
\alpha(d j, d k) \cdot d l:= & \operatorname{tr} B_{\alpha}(d j, d k) \cdot B_{d l}+\operatorname{tr} C_{\alpha}(d j, d k) \cdot C_{d l} \\
& +\operatorname{tr} C_{\alpha}(d j, d k) \cdot C_{d l} .
\end{aligned}
$$

Lising now (5.20) and ( 7.1$)$, it is easy to see that the internal constitutive law $F$ admits an integral representation by a force density $P_{F}$, which depends on ( the coefficients of ) the stress form $\alpha$. Indeed, to this end we have to solve the equation

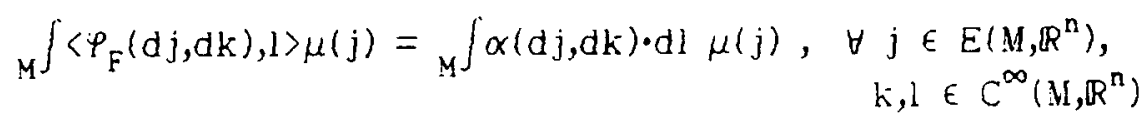

Before doing so, however, we point out that $\alpha$ in $(7.5)$ is not uniquely determined. In fact we have (cf. $[B i, 4]$ ). 
Theorem 7.1 :

Given $\alpha \in A^{1}\left(M, \mathbb{R}^{n}\right)$ and $j \in E\left(M, \mathbb{R}^{n}\right)$ with dh as the integrable part of $\alpha$ then

$$
\int \alpha \cdot d k \mu(j)=\int d h \cdot d k \mu(j),
$$

expressing the fact that the non-integrable part $\beta$ of $\alpha=\mathrm{dh}+\beta$ is orthogonal to $\left.C^{\infty}\left(M, \mathbb{R}^{n}\right)\right|_{\mathbb{R}^{n}}$, regarded as a subspace of $A^{1}\left(M, \mathbb{R}^{n}\right)$.

\section{Proof :}

By Proposition 6.1 we have, $\forall X \in \Gamma T M$,

$$
\begin{aligned}
& \operatorname{dh} X=\sum_{s=1}^{n} m(j)\left(V_{s}, X\right) e_{s}, \\
& \beta(X)=\sum_{s=1}^{n} m(j)\left(Y_{s}^{0}, X\right) e_{s},
\end{aligned}
$$

where $v_{s}=\operatorname{grad}_{j} \tau_{s}, \operatorname{div}_{j} Y_{s}^{0}=0, s=1, \ldots, n$. Then from $(6.24),(6.26)$ and (6.29) it follows

$$
\left(B_{d h}+C_{d h}\right) X=\sum_{s=1}^{n} m(j)\left(V_{s}, X\right) Y_{s}
$$

and

$$
\left(B_{\beta}+C_{\beta}\right) X=\sum_{s=1}^{n} m(j)\left(Y_{s}^{0}, X\right) Y_{s} .
$$

where $Y_{s}=V_{s}+Y_{s}^{0}, s=1, \ldots, n$, satisfies the equation

Hence

$$
m(j)\left(Y_{s}, X\right)=\left\langle\alpha(X), e_{s}\right\rangle, \quad \forall X \in \Gamma T M .
$$

$$
\left(\mathrm{B}_{d h}+C_{d h}\right) o\left(B_{\beta}+C_{\beta}\right) X=\sum_{s=1}^{n} \sum_{s^{\prime}=1}^{n} m(j)\left(Y_{s}^{0}, X\right) m(j)\left(V_{s^{\prime}}, i_{s}\right) i_{s^{\prime}} \cdot
$$

Therefore if $\bar{e}_{1}, \ldots, \bar{e}_{n-1}$ is an orthonormal moving frame on $M$

Next we form

$$
\begin{aligned}
\operatorname{tr}\left(B_{d h}\right. & \left.+C_{d h}\right) \circ\left(B_{\beta}+C_{\beta}\right)=\operatorname{tr}\left(B_{d h} \circ B_{\beta}+C_{d h} \circ C_{\beta}\right) \\
& =\sum_{r=1}^{n-1} \sum_{s^{\prime}=1}^{n} \sum_{s=1}^{n} m(j)\left(Y_{s}^{0}, \bar{e}_{y}\right) m(j)\left(V_{s^{\prime}}, Y_{s}\right) m(j)\left(Y_{s^{\prime}}, \bar{e}_{r}\right) \\
& =\sum_{s=1}^{n} m(j)\left(Y_{s}^{0}, V_{s}\right) .
\end{aligned}
$$

$$
\left\{\begin{array}{l}
c_{d h} d j X=\sum_{s=1}^{n} m(j)\left(V_{s}, X\right) \cdot\left\langle e_{s}, N(j)\right\rangle \cdot N(j) \\
c_{d h} N(j)=\sum^{n}\left\langle e_{s}, N(j)\right\rangle \cdot d j V_{s} .
\end{array}\right.
$$


Similar equations hold for $c_{\beta}$ with $V_{s}$ replaced by $Y_{s}^{0}$ for each $s$. Hence

$$
\left\{\begin{array}{l}
c_{d h} \circ c_{\beta} d j X=\sum_{s^{\prime}=1}^{n} \sum_{s=1}^{n} m(j)\left(Y_{s}^{0}, X\right) \cdot\left\langle e_{s^{\prime}}, N(j)\right\rangle \cdot\left\langle e_{s^{\prime}}, N(j)\right\rangle \cdot V_{s}, \\
c_{d h} \circ c_{\beta} N(j)=\sum_{s^{\prime}=1}^{n} \sum_{s=1}^{n} m(j)\left(V_{s^{\prime}}, Y_{s^{\prime}}\right) \cdot\left\langle e_{s^{\prime}}, N(j)\right\rangle \cdot\left\langle e_{s^{\prime}}, N(j)\right\rangle \cdot N(j) .
\end{array}\right.
$$

Therefore

$$
\operatorname{tr} c_{d h} \circ c_{\beta}=2 \cdot \sum_{s=1}^{n} m(j)\left(V_{s}^{-0}, V_{s}\right)
$$

and hence

$$
\operatorname{tr}\left(B_{d h} \circ B_{\beta}+C_{d h} \circ C_{\beta}+c_{d h} \circ c_{\beta}\right)=3 \cdot \sum_{s=1}^{n} m(j)\left(Y_{s}^{0}, v_{s}\right) .
$$

But (7.14) implies

$$
M \int d h \cdot \beta \mu(j)=3 \cdot \sum_{s=1}^{n} \int m(j)\left(Y_{s}^{0}, V_{s}\right) \mu(j)=0
$$

since $\mathrm{Y}_{\mathrm{s}}^{0}$ and $\mathrm{V}_{\mathrm{s}}$ are $\mathrm{L}_{2}$-orthogonal, $\mathrm{s}=1, \ldots, \mathrm{n}$.

Let us now turn back to $(7.5)$.

At first we study the equation

$$
\left\langle\varphi_{F}, 1\right\rangle=\operatorname{tr}\left(\mathrm{B}_{\alpha} \mathrm{oB}_{d l}+\mathrm{C}_{\alpha} \mathrm{oC}_{\mathrm{dl}}+\mathrm{c}_{\alpha} \mathrm{oc}_{\mathrm{dl}}\right),
$$

where for the sake of simplicity we omited the arguments of $\alpha$ and $P_{F}$. On one hand we note that $1 \in C^{\infty}\left(M, \mathbb{R}^{n}\right)$ can be represented as

$$
i=d j Z_{1}+\Theta_{1} N(j)
$$

and that it will be sufficient to consider only those! for which $\Theta_{1}=1$, i.e.

$$
1=\mathrm{dj} X_{1}+\mathrm{N}(\mathrm{j}) \text {. }
$$

On the other hand according to Proposition 6.t we have for a fixed orthonormal moving frame $e_{1}, \ldots, e_{n-1} \in \Gamma T M$

$$
\begin{aligned}
\operatorname{tr} B_{\alpha} \circ B_{d l}= & \sum_{s=1}^{n-1} \frac{1}{2} m(j)\left(\left(B_{\alpha} \circ(\nabla(j)) Z_{1}+\tilde{\nabla}(j) Z_{l}\right) e_{s}, e_{s}\right) \\
& +\sum_{s=1}^{n-1} m(j)\left(B_{\alpha} \circ W(j) e_{s}, e_{s}\right) \\
= & \sum_{s=1}^{n-1} m(j)\left(B_{\alpha} \circ \nabla(j) e_{s} X_{1}, e_{s}\right)-\sum_{s=1}^{n-1}\left\langle N(j), s(j)\left(e_{s}, B_{\alpha} e_{s}\right)\right. \\
= & \operatorname{div}_{j} B_{\alpha} X_{1}-m(j)\left(\operatorname{div} B_{\alpha}, X_{l}\right) \\
& +\left\langle\operatorname{tr}\left(B_{\alpha} \circ W(j)\right) \cdot N(j), N(j)\right\rangle .
\end{aligned}
$$


We calculate next in the same way $\operatorname{tr} \mathrm{C}_{\alpha}{ } \mathrm{C}_{\mathrm{dl}}$ and obtain, using the fact that

$$
\begin{aligned}
& \operatorname{tr} W(j) \circ C_{\alpha}=0, \\
& \operatorname{tr} C_{\alpha} \circ C_{d l}=\operatorname{div}_{j} C_{\alpha} X_{I}-m(j)\left(\operatorname{div}_{j} C_{\alpha}, X_{l}\right) .
\end{aligned}
$$

It remains now to calculate $\operatorname{tr} c_{\alpha}{ }^{\circ} c_{d l}$. Recalling to this purpose that

$$
c_{d l} \text { dj } Y=S(j)\left(X_{1}, Y\right), \quad \forall Y \in \Gamma T M,
$$

and writing $c_{\alpha} N(j)$ with the help of a field, say $U_{\alpha}$, in $\Gamma$ TM as

$$
c_{\alpha} N(j)=d j U_{\alpha}
$$

we then get

$$
\begin{aligned}
\operatorname{tr} c_{\alpha} \circ c_{d l} & =\sum_{s=1}^{n-1}\left\langle c_{\alpha} \circ c_{d l} d j e_{s}, d j e_{s}\right\rangle+\left\langle c_{d l} o c_{\alpha} N(j), N(j)\right\rangle \\
& =\sum_{s=1}^{n-1}\left\langle c_{\alpha} s(j)\left(X_{l}, e_{s}\right), d j e_{s}\right\rangle+\left\langle c_{d l} d j U_{\alpha}, N(j\rangle\right\rangle \\
& \left.=-\sum_{s=1}^{n-1} m(j)\left(W(j) x_{l}, e_{s}\right): r_{\alpha} N(j), d j e_{s}\right\rangle \\
& -m_{i}(j)\left(W(j) U_{\alpha}, X_{l}\right) \\
= & -\sum_{s=1}^{n-1} m(j)\left(W(j) X_{l}, e_{s}\right) \cdot m(j)\left(U_{\alpha}, e_{s}\right)-m(j)\left(W(j) U_{\alpha}, X_{l}\right) \\
= & -2 m(j)\left(W(j) U_{\alpha}, X_{l}\right) .
\end{aligned}
$$

The equation for $P_{F}$ becomes then

$$
\begin{aligned}
\operatorname{M}_{M}\left\langle\varphi_{f}, 1\right\rangle \mu(j)= & \int\left[\operatorname{div}_{j}\left(\left(B_{\alpha}+C_{\alpha}\right) X_{1}\right)\right. \\
& -\left\langle\operatorname{dj}\left(\operatorname{div}_{j}\left(B_{\alpha}+C_{\alpha}\right)+2 W(j) U_{\alpha}\right)\right. \\
& \left.\left.+\operatorname{tr}\left(B_{\alpha}{ }^{o W}(j)\right) N(j), 1\right\rangle\right] \mu(j) .
\end{aligned}
$$

Using Gauss' theorem this equation yields

Proposition 7.2 :

Let $\dot{F}_{\mathbb{R}^{n}}$ admits an integral representation given by

where the stress form

$$
F_{\mathbb{R}^{n}}(d k)(d j, d l)={ }_{M} \int \alpha(d j, d k) \cdot d l \mu(j), \quad \forall j \in E\left(M, \mathbb{R}^{n}\right),
$$

$$
\alpha:\left.\operatorname{TE}\left(M, \mathbb{R}^{n}\right)\right|_{\mathbb{R}^{n}} \longrightarrow A^{1}\left(M, \mathbb{R}^{n}\right)
$$

splits into

$$
\alpha=c_{\alpha} \cdot d j+d j \cdot C_{\alpha}+d j \cdot B_{\alpha} .
$$

Then $F$ admits an integral representation with a force density $\varphi_{F}$ given at (dj,dk) by 


$$
\begin{aligned}
P(d j, d k)= & -d j\left(\operatorname { d i v } \left(B_{\alpha}(d j, d k)+C_{\alpha}(d j, d k)\right.\right. \\
& \left.+2 W(j) U_{\alpha}(d j, d k)\right)+\operatorname{tr}\left(B_{\alpha}(d j, d k) o w(j)\right) \cdot N(j) .
\end{aligned}
$$

Since $\alpha$ in (7.1) can be replaced by its integrable part as expressed in (7.6) a redundancy occurs in (7.23). Let us therefore rewrite this equation by replacing $\alpha(d j, d k)$ by its integrable part $d h(d j, d k)$. To this end we first rewrite $U_{d h}(d j, d k)$ in terms of $h(d j, d k)$ as done by $[B i, 5]$ and restated in the following :

\section{Lemma 7.3 :}

Let $h=d j X_{h}+\Theta_{h} \cdot N(j)$ for any $h \in C^{\infty}\left(M, \mathbb{R}^{n}\right)$ then $U_{d h}$, as defined in $(7.23)$ takes the form

$$
U_{d h}=W(j) Y_{h}-\operatorname{grad}_{j} \Theta_{h}
$$

Proof :

Equation ( 7.23$)$ reads in the case under consideration as

$$
c_{d h} N(j)=d j U_{d h} .
$$

By (6.44) we have moreover

$$
\begin{aligned}
c_{d h} \operatorname{dj} X & =S(j)\left(X_{h}, X\right)+d \Theta_{h}(X) \cdot N(j) \\
& =-m(j)\left(W(j) X_{h}-\operatorname{grad}_{j} \Theta_{h}, X\right) \cdot N(j) .
\end{aligned}
$$

Therefore

$$
\begin{aligned}
c_{d h}^{2} N(j) & =c_{d h} \cdot d j U_{d h} \\
& =-m(j)\left(W(j) K_{h}-\operatorname{grad}_{j} \Theta_{h}, U_{d h}\right) N(j)
\end{aligned}
$$

implying (7.26).

In view of $(7.6)$ and $(7.26)$ we obtain

\section{Corollary 7.8 :}

Since for $\left.a n y(d j, d h) \in T E\left(M, \mathbb{R}^{n}\right)\right|_{\mathbb{R}^{n}}$ the stress-form $x$ splits uniquely into

$$
\alpha(d j, d k)=d h(d j, d k)+\beta(d j, d k)
$$

with $h(d j, d h)$ the integrable and $\beta(d j, d k)$ the non-integrable part respectively, equation $(7.25)$ turns into

$$
\begin{aligned}
\rho(d j, d k)= & -\operatorname{div} B_{d h}(d j, d k)+C_{d h}(d j, d k) \\
& +2 W(j)^{2} X_{h}-2 W(j) \operatorname{grad}_{j} \Theta_{h}
\end{aligned}
$$

where

$$
\mathrm{h}=\mathrm{dj} Z_{\mathrm{h}}+\Theta_{\mathrm{h}} N(\mathrm{j})
$$

with $X_{h} \in \Gamma T M$ and $\Theta_{h} \in C^{\infty}(M, \mathbb{R})$.

Using now the equations of motion (3.25) of the deformable medium $M$ we 
immediately obtain the following

\section{Main theorem :}

Let $M$ be a moving deformable medium of codimension 1 subjected to an internal constitutive law

$$
F: C^{\infty}\left(M, \mathbb{R}^{\mathrm{n}}\right) \times E\left(M, \mathbb{R}^{\mathrm{n}}\right) \times C^{\infty}\left(M, \mathbb{R}^{\mathrm{n}}\right) \longrightarrow \mathbb{R},
$$

with

and

$$
F=d^{*} F_{\mathbb{R}^{n}}
$$

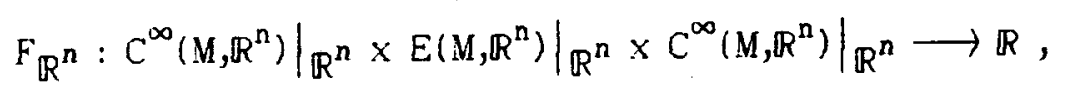

a smooth map admitting an integral representation given by the so-called stress form

$$
\alpha:\left.\operatorname{TE}\left(M, \mathbb{R}^{\mathrm{n}}\right)\right|_{\mathbb{R}^{n}} \longrightarrow A^{1}\left(M, \mathbb{R}^{\mathrm{n}}\right)
$$

This stress form $\alpha$ decomposes according to (6.34) at each

into

$$
\left.(d j, d k) \in T E\left(M, \mathbb{R}^{n}\right)\right|_{\mathbb{R}^{n}}=\left.\left.E\left(M, \mathbb{R}^{n}\right)\right|_{\mathbb{R}^{n} \times C^{\infty}\left(M, \mathbb{R}^{n}\right)}\right|_{\mathbb{R}^{n}}
$$

$$
\alpha(d j, d k)=c_{\alpha}(d j, d k) \cdot d j+d j \cdot C_{\alpha}(d j, d k)+d j \cdot B_{\alpha}(d j, d k)
$$

The equation of motion on $E\left(M, \mathbb{R}^{\mathrm{n}}\right)$ described by a smooth curve

$$
\sigma:(-\lambda, \lambda) \longrightarrow E\left(M, \mathbb{R}^{\mathrm{n}}\right), \quad \lambda>0,
$$

is then giren by

$$
\begin{aligned}
M_{M} \rho(\sigma(t))\langle\tilde{\sigma}(t), 1\rangle \mu(\sigma(t)) & ={ }_{M} \int \alpha(d \sigma(t), d \dot{\sigma}(t)) \cdot d l \mu(\sigma(t)) \\
& =\int_{M} \int\left\langle P_{F}(d \sigma(t), d \dot{\sigma}(t)), 1\right\rangle \mu(\sigma(t)),
\end{aligned}
$$

where the force density $\rho_{F}$ satisfies (7.25). Using the fact that (7.29) implies

$$
\rho(\sigma(t)) \ddot{\sigma}(t)=P_{F}(d \sigma(t), d \dot{\sigma}(t)), \quad \forall t \dot{E}(-\lambda, \lambda)
$$

and writing $\dot{\sigma}(t)$ in $\mathbb{R}^{\mathrm{n}}$ as

$$
\dot{\sigma}(t)=d \sigma(t) Z(t)+\epsilon(\sigma(t), \dot{\sigma}(t)) N(\sigma(t)), \quad \forall t \in(-\lambda, \lambda),
$$

where $Z(t) \in \Gamma T M$ and $\epsilon(\sigma(t), \dot{\sigma}(t)) \in C^{\infty}(M, \mathbb{R})$, the equation of motion (7.30) splits into the coupled system 


$$
\left\{\begin{aligned}
& \nabla(\sigma(t))_{Z(t)} Z(t)+Z(t)+2 \epsilon(\sigma(t), \dot{\sigma}(t)) W(\sigma(t)) Z(t) \\
&-\operatorname{grad}_{\sigma(t)} \epsilon(\sigma(t), \dot{\sigma}(\mathrm{t})) \\
&\left.=\rho^{-1}(\sigma(t))\left[-\operatorname{div}_{\sigma(t)} T_{\alpha}(\mathrm{d} \sigma(\mathrm{t}), \mathrm{d} \dot{\sigma}(\mathrm{t}))-2 W(\sigma(t)) U_{\alpha}\right)\right], \\
& \dot{\epsilon}(\sigma(\mathrm{t}), \dot{\sigma}(\mathrm{t}))=\rho^{-1}(\sigma(\mathrm{t})) \operatorname{tr} \mathrm{B}_{\alpha}(\mathrm{d} \sigma(\mathrm{t}), \mathrm{d} \dot{\sigma}(\mathrm{t})) W(\sigma(\mathrm{t})) \\
&-\mathrm{d} \epsilon(\sigma(\mathrm{t}), \dot{\sigma}(\mathrm{t})) Z(\mathrm{t})+\hat{h}(\sigma(\mathrm{t}))(Z(\mathrm{t}), Z(\mathrm{t})),
\end{aligned}\right.
$$

where the smooth two tensor $T_{\alpha}(\mathrm{dj}, \mathrm{dk})$, the so-called stress tensor, is defined by

$$
\left.T_{\alpha}(d j, d k)(X, Y)=m(j)\left(B_{\alpha}+C_{\alpha}\right)(d j, d k) X, Y\right), \quad \forall X, Y \in \Gamma T M
$$

and $h(\sigma(t))$ denotes the second fundamental form of $\sigma(t)$.

\section{Remark :}

(i.31) corresponds to Cauchy's law in the mechanics of continua.

Using Corollary 7.8 equations (7.31) turn into

\section{Corollary :}

Using the splitting of the stress-form into an integrable dh and a non-integrable part $\beta$ respectively, $(i .31)$ reads

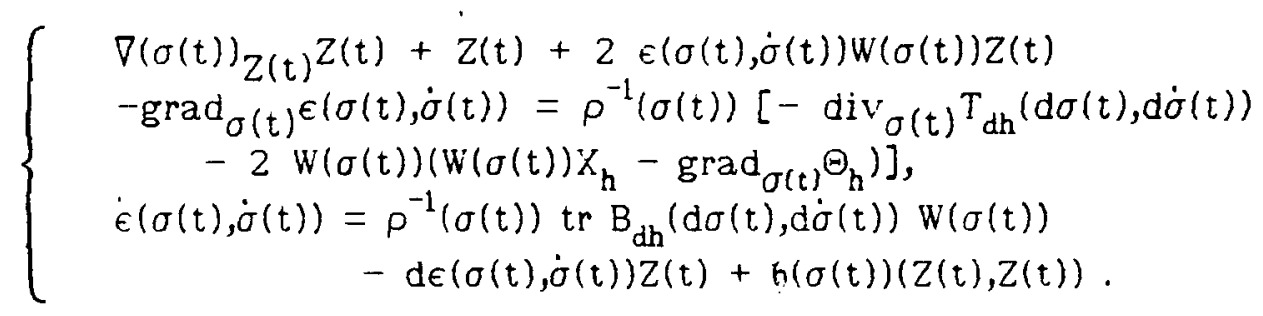

Example 7.9 :

Let $\alpha$ be given by

$$
\alpha(d j, d k)=-\tau(d j, d k) \cdot d j,
$$

$\forall j \in E\left(M, \mathbb{R}^{\mathrm{n}}\right), k \in C^{\infty}\left(M, \mathbb{R}^{\mathrm{n}}\right)$,

where

$$
\tau:\left.T E\left(M, \mathbb{R}^{n}\right)\right|_{\mathbb{R}^{n}} \longrightarrow C^{\infty}\left(M, \mathbb{R}^{n}\right)
$$

is a smooth map. Then we have

$$
\left\{\left.\begin{array}{l}
B_{\alpha}(d j, d k)=\tau(d j, d k) \cdot I d_{T M}, \\
C_{\alpha}(d j, d k)=0, \\
c_{\alpha}(d j, d k)=0 .
\end{array} \quad \forall(d j, d k) \in T E\left(M, \mathbb{R}^{n}\right)\right|_{\mathbb{R}^{n}}\right.
$$


Therefore the stress tensor $T_{\alpha}$ and the force density $P_{F}$ become

$$
T_{\alpha}(\mathrm{dj}, \mathrm{dk})(X, Y)=\tau(\mathrm{dj}, \mathrm{dk}) \cdot \mathrm{m}(\mathrm{j})(X, Y), \quad \forall X, Y \in \Gamma T M,
$$

and

$$
\varphi_{F}(d j, d k)=d j \operatorname{grad} \tau(d j, d k)+\tau(d j, d k) \cdot H(j) N(j)
$$

Then the Main theorem reduces to the following

\section{Proposition 7.10 :}

Let the hypotheses of the Main theorem hold and assume that $\alpha$ is given by (7.34). Then the equations of motion of the deformable medium $M$ are given by

$$
\begin{aligned}
& \nabla(\sigma(t)) Z(t) Z(t)+Z(t)+2 \cdot \epsilon(\sigma(t), \dot{\sigma}(t)) W(\sigma(t)) Z(t) \\
& -\operatorname{grad}_{\sigma(t)} \epsilon(\sigma(t), \dot{\sigma}(\mathrm{t}))=\rho^{-1}(\sigma(\mathrm{t})) \operatorname{grad}_{\sigma(\mathrm{t})} \tau(\mathrm{d} \sigma(\mathrm{t}), \mathrm{d} \dot{\sigma}(\mathrm{t})), \\
& \dot{\epsilon}(\sigma(t), \dot{\sigma}(t))=\rho^{-1}(\sigma(t)) \tau(d \sigma(t), d \dot{\sigma}(t)) \cdot H(\sigma(t)) \\
& -\mathrm{d} \epsilon(\sigma(t), \dot{\sigma}(t)) Z(t)+h(\sigma(t))(Z(t), Z(t)) \text {. }
\end{aligned}
$$

We call ( 7.39$)$ the equations of motion of a perfect deformable medium. The corresponding constitutive law will be refered too as the perfect constitutive law.

The above corollary relates with the motion induced by a reduced constitutive law introduced earlier as shown by the following

\section{Corollary 7.11 :}

Let $F$ be a smooth $\mathbb{R}^{n}$-invariant constitutive law splitting into the sum

$$
F=F^{0}+F^{\prime},
$$

where $F^{0}$ and $F^{\prime}$ are smooth $\mathbb{R}^{n}$-invariant constitutive laws admitting both stress forms $\alpha^{0}$ and $\alpha$ respectively. Assume moreover that $\alpha^{0}$ and $\alpha$ have the decompositions

$$
\left\{\begin{array}{c}
\alpha^{0}(d j, d k)=\tau(d j, d k) \cdot d j, \\
\alpha(d j, d k)=c_{\alpha}(d j, d k) \cdot d j+d j \cdot C_{\alpha}(d j, d k)+d j \cdot B_{\alpha}{ }^{\prime}(d j, d k), \\
\left.\forall(d j, d k) \in T E\left(M, \mathbb{R}^{n}\right)\right|_{\mathbb{R}^{n}}
\end{array}\right.
$$

with

$$
\operatorname{tr} B_{\alpha}^{\prime}(d j, d k)=0
$$

Then the motion

$$
\sigma:(-\lambda, \lambda) \longrightarrow E\left(M, \mathbb{R}^{\mathrm{n}}\right)
$$

induced by $F$ satisfies the equations 


$$
\begin{aligned}
& \nabla(\sigma(t))_{Z(t)} Z(t)+\dot{Z}(t) \\
& +2 \epsilon(\sigma(t), \dot{\sigma}(t)) w(\sigma(t)) Z(t)-\operatorname{grad}_{\sigma(t)} \epsilon(\sigma(t), \dot{\sigma}(t)) \\
& =\rho^{-1}(\sigma(t))\left[\operatorname{grad}_{\sigma(t)} \tau(\mathrm{d} \sigma(\mathrm{t}), \mathrm{d} \dot{\sigma}(\mathrm{t}))-\mathrm{div}_{\sigma(\mathrm{t})}\right)_{\alpha}^{\prime}(\mathrm{d} \sigma(\mathrm{t}), \mathrm{d} \dot{\sigma}(\mathrm{t})) \\
& \left.\left.-2 \mathrm{~W}(\sigma(\mathrm{t})) \mathrm{U}_{\alpha}\right)\right] \\
& \dot{\epsilon}(\sigma(t), \dot{\sigma}(t))=\rho^{-1}(\sigma(t))\left[\operatorname{tr}\left(B_{\alpha}^{\prime}(d \sigma(t), d \dot{\sigma}(t)) \cdot W(\sigma(t))\right)\right. \\
& \text { - } \tau(\mathrm{d} \sigma(\mathrm{t}), \mathrm{d} \dot{\sigma}(\mathrm{t})) \mathrm{H}(\sigma(\mathrm{t}))] \\
& -d \epsilon(\sigma(t), \dot{\sigma}(t)) Z(t)+h(\sigma(t))(Z(t), Z(t)) \text {. }
\end{aligned}
$$




\section{The structural viscosity}

As known the notion of viscosity was first introduced by Newton as "the resistance which arises from the lack of slipperiness of the parts of the liquid". He made the assumption that the viscosity "is proportional to the velocity with which the parts of the liquid are separated from one another". As a measure of the viscous resistance one has introduced the coefficient of viscosity $v$.

In this section we introduce the notion of structural viscosity, as done in $[\mathrm{Bi}, 5]$, i.e. the notion of viscosity within our apparatus.

Let again $M$ be a moving deformable medium of codimension 1 and assume that its motion is due only to an internal constitutive law $F$. As shown $F$ is $\mathbb{R}^{\mathrm{n}}$-invariant and admits the representation

with

$$
F=d^{*} F_{\mathbb{R}^{n}}
$$

$$
F_{\mathbb{R}^{n}}:\left.\left.C^{\infty}\left(M, \mathbb{R}^{n}\right)\right|_{\mathbb{R}^{n} \times E\left(M, \mathbb{R}^{n}\right)}\right|_{\mathbb{R}^{n}} \times\left. C^{\infty}\left(M, \mathbb{R}^{n}\right)\right|_{\mathbb{R}^{n}} \longrightarrow \mathbb{R}
$$

a smooth map. In addition we assume that $F_{\mathbb{R}^{n}}$ admits a stress form

$$
\alpha:\left.\operatorname{TE}\left(M, \mathbb{R}^{n}\right)\right|_{\mathbb{R}^{n} \longrightarrow A^{1}\left(M, \mathbb{R}^{n}\right)}
$$

According to Proposition 6.1 the $\mathbb{R}^{\mathrm{n}}$-valued one-form $\propto$ admits the decomposition

$$
\alpha(d j, d k)=d h(d j, d k)+\beta(d j, d k),
$$

where the integrable part $h \in C^{\infty}\left(M, \mathbb{R}^{n}\right)$ is uniquely determined up to a constant. Next we give $h$ and $k$ the equivalent forms

$$
h(d j, d k)=d j X_{h}(d j, d k)+h^{1}(d j, d k)
$$

and respectively

$$
k=\mathrm{dj} \chi_{\mathrm{x}}+\mathrm{k}^{\perp} \text {. }
$$

Moreoter we set, using the fact that $M$ has codimension 1 ,

$$
h^{\perp}(d j, d k)=\Theta_{h}(d j, d k) N(j)
$$

We split now according to Hodge's theorem $X_{\mathrm{h}}$ and $Y_{\mathrm{x}}$ into

and

$$
\begin{aligned}
& X_{\mathrm{h}}=X_{\mathrm{h}}^{0}+\operatorname{grad}_{\mathrm{j}{ }_{\mathrm{h}}}, \\
& \operatorname{div}_{\mathrm{j}} X_{\mathrm{h}}^{0}=0,
\end{aligned}
$$

$$
\begin{aligned}
& X_{k}=y_{k}^{0}+\operatorname{grad}_{j} \Psi_{k}, \\
& \operatorname{div}_{j} X_{k}^{0}=0
\end{aligned}
$$


respectively, Let us remind of proposition 6.2, expressing in particular that $x_{h}^{0}$ and $x_{k}^{0}$ are uniquely determined by $\alpha$ and $d k$ respectively.

We relate next $X_{h}^{0}$ and $X_{k}^{0}$ uniquely to each other by setting

$$
\chi_{h}^{0}(d j, d k)=v(d j, d k) X_{k}^{0}+\hat{Y}_{h}(d j, d k),
$$

where $v(\mathrm{dj}, \mathrm{dk}) \in C^{\infty}(M, \mathbb{R})$ and $\hat{X}_{\mathrm{h}}(\mathrm{dj}, \mathrm{dk}) \in \Gamma T M$ is pointwise orthogonal to $X_{k}^{0}$, and call the function $v(\mathrm{dj}, \mathrm{dk})$ coefficient of structural viscosity.

Accordingly we call the deformable media, whose constitutive laws depend only on $\mathrm{k}^{1}$, frictionless deformable media, while the deformable media whose constitutive laws depend on the whole of $k$, will be called frictional ones. Taking now into account (8.1) and (8.2), Proposition 6.4 becomes

\section{Proposition 8.1 :}

Let $\alpha \in A^{1}\left(M, \mathbb{R}^{n}\right)$ and $j \in E\left(M, \mathbb{R}^{n}\right)$ be given and assume that $M$ has codimension 1 . Then the following relations hold:

$$
\left\{\begin{array}{l}
\alpha=d h+\beta, \\
\alpha(X)=c_{\alpha} d j X+d j C_{\alpha} Z+d j B_{\alpha} X, \quad \forall X \in \Gamma T M, \\
h(d j, d k)=d j X_{h}^{0}(d j, d k)+d j \operatorname{grad}_{j} \psi_{h}+\Theta_{h}(d j, d h) N(j), \\
\operatorname{div} X_{\cdot h}^{0}=0, \\
X_{k}=X_{k_{k}}^{0}+\operatorname{grad}_{j} \psi_{k}, \\
\operatorname{div}_{j} X_{h}^{0}=0, \\
X_{h}^{0}=v(d j, d k) X_{k}^{0}+\hat{X}_{k}(d j, d k),
\end{array}\right.
$$

$$
\begin{aligned}
& c_{\alpha}(d j, d k) \cdot d j=\left(d h^{\perp}(d j, d k)\right)^{\perp}+v(d j, d k) S(j)\left(X_{-k}^{0}, \ldots\right) \\
& +S(j)(\hat{K}, .)+S(j)\left(\operatorname{grad}_{j} \psi_{h}, \ldots\right)+c_{\beta}(d j, d k) \cdot d j, \\
& C_{\alpha}(\mathrm{dj}, \mathrm{dk})=\frac{1}{2}\left[\nabla(\mathrm{j}) v(\mathrm{dj}, \mathrm{dk}) \gamma_{\mathrm{k}}^{0}-\tilde{\nabla}(\mathrm{j}) v(\mathrm{dj}, \mathrm{dk}) \gamma_{\mathrm{k}}^{0}\right] \\
& +\frac{1}{2}\left[\nabla(j) \hat{\psi}_{h}(d j, d k)-\tilde{\nabla}(j) \hat{\psi}_{h}(d j, d k)\right]+\sigma_{\beta}(1, j, d k), \\
& B_{\alpha}(d j, d k)=\frac{1}{2} L_{v}(d j, d k) Z_{k}^{0}+\frac{1}{2} L_{Z_{k}}(d j, d h) \\
& +\frac{1}{2} L_{\operatorname{grad}_{j} \psi_{h}(d j, d k)}+\Theta_{h}(d j, d k) W(j)+B_{\beta}(d j, d k), \\
& \left.\forall(d j, d k) \in T E\left(M, \mathbb{R}^{n}\right)\right|_{\mathbb{R}^{n}} .
\end{aligned}
$$

If now $v(d j, d k)$ is a constant map in $C^{\infty}(M, \mathbb{R})$, then we get

$$
\begin{aligned}
& C_{\alpha}(d j, d k)=\frac{1}{2} v(d j, d k)\left(\nabla(j) z_{k}^{0}-\tilde{\nabla}(j) \gamma_{k}^{0}\right) \\
& +\frac{1}{2}\left[\nabla(j) \hat{X}_{h}(d j, d k)-\tilde{\nabla}(j){\hat{\psi_{h}}}_{h}(d j, d k)\right]+C_{\beta}(d j, d k), \\
& \mathrm{B}_{\alpha}(\mathrm{dj}, \mathrm{dk})=\frac{1}{2} v(\mathrm{dj}, \mathrm{dk}) \mathrm{L}_{X_{\mathrm{k}}} \mathrm{l}+\frac{1}{2} \mathrm{~L}_{\mathrm{X}_{\mathrm{h}}}(\mathrm{dj}, \mathrm{dk}) \\
& +\frac{1}{2} L_{\operatorname{grad} j \psi_{h}(d j, d k)}+\Theta_{h}(d j, d k) w(j)+B_{\beta}(d j, d k), \\
& \left.\forall(d j, d k) \in T E\left(M, \mathbb{R}^{n}\right)\right|_{\mathbb{R}^{n}} .
\end{aligned}
$$


Lsing now the definition $(\bar{i} .32)$ of the stress tensor we get

(8.8)

$$
\begin{aligned}
T_{\alpha}(d j, d k)(X, Y) & =m(j)\left(\left(\frac{1}{2} v(d j, d k) L_{X_{k}^{0}}+\frac{1}{2} v(d j, d k)\right.\right. \\
& \left(\nabla(j) X_{k}^{0}-\tilde{\nabla}(j) X_{k}^{0}\right)+\frac{1}{2} L_{\hat{X}_{h}}(d j, d k) \\
& +\frac{1}{2}\left[\nabla(j) \hat{X}_{h}(d j, d k)-\tilde{\nabla}(j) \hat{Z}_{h}(d j, d k)\right] \\
& -\frac{1}{2} L_{g r a d j} \psi_{h}(d j, d k)+\Theta_{h}(d j, d k) \cdot W(j) \\
& \left.\left.+B_{\beta}(d j, d k)+C_{\beta}(d j, d k)\right) X, Y\right) .
\end{aligned}
$$

It is this equation which motivates us to call the function $v(\mathrm{dj}, \mathrm{dh})$ coefficient of structural viscosity. 
Let us suppose that the motion of the deformable medium $\mathrm{M}$ is governed by the smooth constitutive law

$$
F: C^{\infty}\left(M, \mathbb{R}^{n}\right) \times E\left(M, \mathbb{R}^{n}\right) \times C^{\infty}\left(M, \mathbb{R}^{n}\right) \longrightarrow \mathbb{R}
$$

and assume that $F$ splits into

$$
F=F_{\text {ext }}+F_{\text {int }},
$$

where the internal constitutive law $F_{\text {int }}$ and the external one $F_{\text {ext }}$ are also smooth. As shown $F_{\text {int }}$ is $\mathbb{R}^{n}$-invariant and admits the representation

with

$$
F_{\text {int }}=d^{*} F_{\mathbb{R}^{n}}
$$

$$
F_{\mathbb{R}^{n}}:\left.\left.\left.C^{\infty}\left(M, \mathbb{R}^{n}\right)\right|_{\mathbb{R}^{n} \times E\left(M, \mathbb{R}^{n}\right)}\right|_{\mathbb{R}^{n} \times C^{\infty}\left(M, \mathbb{R}^{n}\right)}\right|_{\mathbb{R}^{n}} \longrightarrow \mathbb{R}
$$

Finally we assume that both $F_{\text {ext }}$ and $F_{\mathbb{R}^{n}}$ admit integral representations and denote by ' $\rho_{\text {ext }}$ and ' $\rho_{\text {int }}$ the corresponding force densities. According to (3.13) and (3.26) we have the splittings

$$
\begin{array}{r}
P_{F_{\text {int }}}(j, k)=d j \operatorname{grad}_{j} \tau_{\text {int }}(j, k)+d j Y_{\text {int }}^{0}(j, k)+P_{F_{\text {int }}}(j, k)^{\perp}, \\
P_{F_{\text {ext }}}(j, k)=d j \operatorname{grad}_{j} \tau_{\text {ext }}(j, k)+d j Y_{\text {ext }}^{0}(j, k)+\varphi_{F_{\text {ext }}}(j, k)^{\perp}, \\
\forall j \in E\left(M, \mathbb{R}^{n}\right), k \in C^{\infty}\left(M, \mathbb{R}^{n}\right),
\end{array}
$$

and hence

$$
P_{F}(j, h)=d j \operatorname{grad}_{j} \tau(j, k)+d j I^{O}(j, k)+P_{F}(j, k)^{\perp}
$$

where

$$
\begin{aligned}
\tau_{\text {int }}(j, h)+ & \tau_{\text {ext }}(j, k)=\tau(j, k), \\
Y_{\text {int }}^{0}(j, k)+ & Y^{0} \text { ext }(j, k)=Y^{0}(j, k), \\
f_{F}^{f}(j, k)^{\perp}+ & P_{F_{\text {ext }}}(j, k)^{\perp}=\varphi_{F}(j, k)^{\perp}, \\
& \forall j \in E\left(M, \mathbb{R}^{n}\right), k \in C^{\infty}\left(U, \mathbb{K}^{n}\right) .
\end{aligned}
$$

Since $\rho_{F_{\text {int }}}$ is $\mathbb{R}^{\mathrm{n}}-$ invariant, its tangential and normal parts grad $\tau_{\text {int }}+\mathrm{Y}^{0}$ int and $P_{F_{\text {int }}}$ respectively are also $\mathbb{R}^{n}$-invariant. Lsing now the $\mathbb{R}^{n}$-invariance of the Laplace-Beltrami operator $\lrcorner$, we infer that $T$ and consequently grad $\tau$ are $\mathbb{R}^{n}$-invariant. Accordingly we split $F_{\text {int }}$ into

$$
F_{\text {int }}=F_{\text {int }}^{0}+F_{\text {int }}^{\prime}
$$


where $F^{0}$ int has the force density $\varphi_{F_{\text {int }}}^{0}$ defined by

$$
\varphi_{F_{\text {int }}}^{0}(j, k)=d j \operatorname{grad}_{j} \tau_{\text {int }}(d j, d k) .
$$

and $F_{\text {int }}^{\prime}$ admits the stress form

$$
\left\{\begin{array}{l}
\alpha: T E\left(M, \mathbb{R}^{n}\right) \rightarrow A^{1}\left(M, \mathbb{R}^{n}\right) \\
\alpha(d j, d k)=c_{\alpha}(d j, d k) \cdot d j+d j c_{\alpha}^{\prime}(d j, d k)+d j B_{\alpha}{ }^{\prime}(d j, d k)
\end{array}\right.
$$

Hence

$$
\begin{aligned}
\alpha(d j, d k)= & c_{\alpha}{ }^{\prime}(d j, d k) \cdot d j+d j C_{\alpha}(d j, d k)+ \\
& d j\left(-\tau_{i n t}(d j, d k) I d_{T M}+B_{\alpha}(d j, d k)\right) .
\end{aligned}
$$

Lising $(7.40)$ we then find

$$
\begin{aligned}
& P_{F_{\text {int }}}(j, k)=\varphi_{F_{\text {int }}}^{0}(j, k)+\varphi_{F_{\text {int }}^{\prime}}^{\prime}(j, k) \\
& =d j \operatorname{grad}_{j} \tau_{\text {int }}(d j, d k)+d j Y_{\text {int }}^{0}(d j, d k)+P_{F_{\text {int }}}(d j, d k)^{\perp} \\
& =d j \operatorname{grad}_{j} \tau_{\text {int }}(d j, d k)-d j\left(d i v_{j} B_{\alpha}^{\prime}(d j, d k)+d i v_{j} C_{\alpha}{ }^{\prime \prime}(d j, d k)\right. \\
& \left.\quad+2 W(j)\left(W(j) Y_{h}-\operatorname{grad} \Theta_{h}\right)\right)+\left[\operatorname{tr}\left(B_{\alpha}(d j, d k) \cdot W(j)\right)-\right. \\
& \left.\quad \tau_{\text {int }}(d j, d k) \cdot H(j)\right] N(j),
\end{aligned}
$$

$$
\begin{aligned}
T_{\alpha}(j, k)(X, Y)= & -\tau_{\text {int }}(d j, d k) m(j)(X, Y) \\
& +m(j)\left(\left(B_{\alpha}{ }^{\prime}+C_{\alpha}{ }^{j}\right)(d j, d k) K, Y\right), \forall X, Y \in \Gamma T M .
\end{aligned}
$$

On the other hand $\alpha$ 'splits according to Proposition 6.4 at (dj.dk) into

$$
\alpha^{\prime}(d j, d k)=d h(d j, d k)+\beta(d j, d k)
$$

where $h(d, d k) \in C^{\infty}\left(\mathrm{k}, \mathbb{R}^{\mathrm{n}}\right)$. Writing

$$
\begin{aligned}
& h(d j, d k)=d j X_{h}(d j, d k)+\Theta_{h}(d j, d k) X(j), \\
& h(d j, d k)=d j X_{k}(d j, d k)+\Theta_{k}(d j, d k) N(j),
\end{aligned}
$$

with $\Theta_{h}, \Theta_{k} \in C^{\infty}(M, \mathbb{R})$, we obtain by $(9.10)$ and Proposition 5.t

$$
\begin{aligned}
P_{F_{\text {int }}}(j, k) & =d j \operatorname{grad}_{j} \tau_{i n t}(d j, d k)-d j\left(j(j) X_{h}\right. \\
& +W(j) \operatorname{grad}_{j} \Theta_{h}(d j, d k)+\Theta_{h}(d j, d k) \operatorname{grad} j(j) \\
& \left.+2 W(j)\left(W(j) K_{h}-\operatorname{grad} \Theta_{h}\right)\right) \\
& +\left(\operatorname{tr}\left(\left[\frac{1}{2} L_{X_{h}(d j, d k)}+\Theta_{h}(d j, d k) W(j)\right] \circ W(j)\right)\right. \\
& \left.-\tau_{\text {int }}(d j, d k) H(j)\right) N(j)
\end{aligned}
$$

Decomposing now $X_{\mathrm{h}}(\mathrm{dj}, \mathrm{dk})$ and $X_{\mathrm{k}}$ by the Hodge theorem into 
(9.13)

$$
\begin{aligned}
& \left\{\begin{array}{l}
X_{h}(d j, d k)=X_{h}^{0}(d j, d k)+\operatorname{grad}_{j} \psi_{h}(d j, d k), \\
\operatorname{div}_{j} X_{h}^{0}=0,
\end{array}\right. \\
& \left\{\begin{array}{l}
X_{k}(d j, d k)=X_{k}^{0}(d j, d k)+\operatorname{grad}_{j} \psi_{k}(d j, d k), \\
\operatorname{div}_{j} X_{k}^{0}=0,
\end{array}\right.
\end{aligned}
$$

(9.14)

introducing the structural viscosity $v(d j, d k) \in C^{\infty}(M, R)$ via

$$
X_{h}^{0}(d j, d k)=v(d j, d k) X_{k}^{0}+\hat{X}_{h}(d j, d k)
$$

and noting that

$$
\frac{1}{2} \operatorname{tr}\left(L_{Z} \cdot W(j)\right)=d i v_{j} W(j) Z-d H(j) Z
$$

from $(9.2),(9.3)$ and $(9.11)$ we obtain

$$
\begin{aligned}
\rho_{F}(j, k) & =P_{F_{e x t}}(j, k)+d j\left(\operatorname{grad}_{j} \tau(j, k)-\Delta(j)\left[v(d j, d k) X_{k}^{0}\right.\right. \\
& \left.+\hat{X}_{k}(d j, d k)+\operatorname{grad}_{j} \psi_{h}(d j, d k)\right]-W(j)\left[\operatorname{grad}_{j} \Theta_{h}(d j, d k)\right. \\
& \left.\left.+2\left(W(j) X_{h}-\operatorname{grad} \Theta_{h}\right)\right]-\Theta_{h}(d j, d k) \operatorname{grad}_{j} H(j)\right] \\
& -\left(\tau_{\text {int }}(d j, d k) H(j)+d H(j)\left[v(d j, d k) X_{k}^{0}+\hat{X}_{h}(d j, d k)\right]\right. \\
& -\operatorname{div} j v(d j, d k) W(j) X_{k}^{0}-\operatorname{div}_{j} W(j) \hat{X}_{h}(d j, d k) \\
& \left.-\Theta_{h}(d j, d k) \operatorname{tr} W(j)^{2}\right) N(j),
\end{aligned}
$$

$$
\begin{aligned}
T_{\alpha}(d j, d k)(X, Y) & =-\tau_{i n t}(d j, d k) m(j)(X, Y) \\
+ & \frac{1}{2}\left(L_{v(d j, d k) X_{k}^{0}}+L_{\hat{X}_{k}(d j, d k)}+L_{g r a d j} U_{h}(d j, d k)\right. \\
& (m(j))(X, Y)+m(j)\left(W_{h}(j) X, Y\right) \\
+ & m(j)\left(C_{i X}{ }^{\prime}(d j, d k) X, Y\right)
\end{aligned}
$$

Consequently we may state the following theorem, based on (9.17), (9.18) and (3.25)

Theorem 9.1:

Let $F: C^{\infty}\left(M, \mathbb{R}^{n}\right) \times E\left(M, \mathbb{R}^{n}\right) \times C^{\infty}\left(M, \mathbb{R}^{n}\right) \rightarrow \mathbb{R}$ be a smooth constitutive law admitting the splitting (9.1), i.e.

$$
F=F_{\text {int }}+F_{\text {ext }} \text {, }
$$

where both the internal and the external constitutive laws, $F_{\text {int }}$ and $F_{\text {ext }}$ respectively, admit integral representations with the respective force densities $P_{F_{\text {int }}}$ and $\rho_{F_{\text {ext }}}$.

Then the general equations of motion of a deformable medium are given by 
(9. 19)

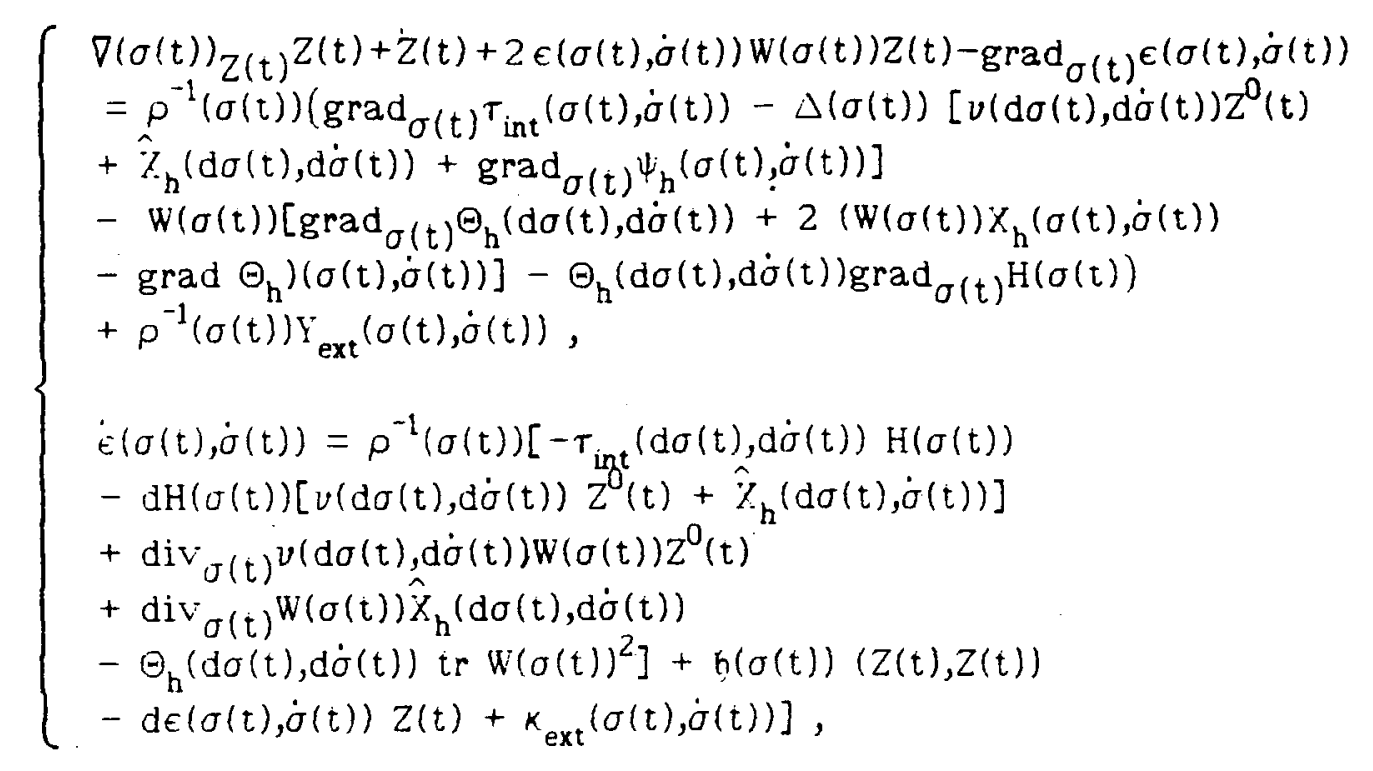

where $\varphi^{\perp}{ }_{\text {ext }}(\sigma(t), \dot{\sigma}(t))=\kappa_{\text {ext }}(\sigma(t), \dot{\sigma}(t)) N(\sigma(t))$.

The motion of a deformable medium along a fixed surface $i(M) \subset \mathbb{R}^{\mathrm{n}}$ is given by

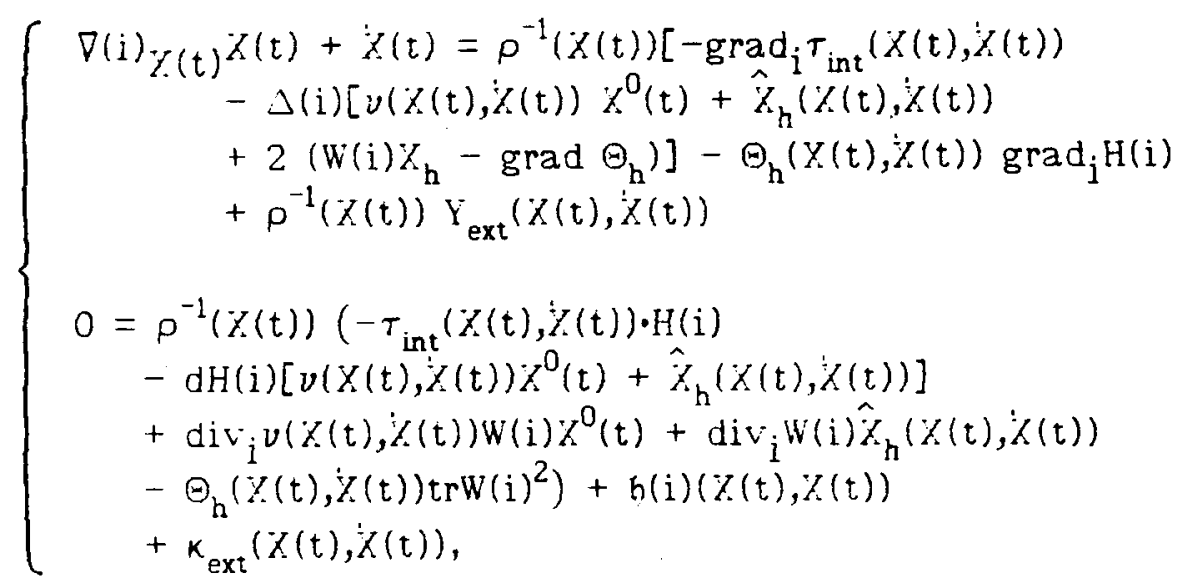

where $Z(t)$ is the push-forward $(4.20)$ of $Z(t)$ by $g(t j \in$ Diff $M$.

As an example let us consider the stress form

$$
\alpha(\mathrm{dj}, \mathrm{dk})=-\tau_{\text {int }}(\mathrm{dj}, \mathrm{dk}) \cdot \mathrm{d} \mathrm{j}+v \mathrm{dj} \cdot \mathrm{L}_{Y_{\mathrm{k}}}
$$

with a constant $v \in \mathbb{R}$. Then the motion along $i(M) \in \mathbb{R}^{n}$ is governed by

$$
\begin{aligned}
& \rho(X(\mathrm{t}))(\nabla(\mathrm{i}) \\
& X(\mathrm{t}) \\
& \left.\quad=-\operatorname{grad}_{\mathrm{i}} \tau_{\text {int }}(\mathrm{g}(\mathrm{t}), X(\mathrm{t}))-v(\mathrm{t})\right) \\
& \quad-\Delta(\mathrm{i}) X(\mathrm{t})-v \cdot \mathrm{Ric}(\mathrm{i}) X(\mathrm{t})
\end{aligned}
$$

in case $\operatorname{div}_{i} X(t)=0$. Thus the equation (9.22), a varier-Stokes type of equation, is an approximation to (9.20).

\section{Remark:}

As done in $[\mathrm{Bi}, 5]$ a type of pressure $\pi(d j, d h)$ can be introduced by forming the $\mathrm{L}^{2}$-component of $\operatorname{tr} \mathrm{B}_{\alpha}(\mathrm{dj}, \mathrm{dk}) \mathrm{W}(\mathrm{j})$ (in $i .25$ ) along $\mathrm{H}(\mathrm{j})$ yielding the decomposition 


$$
B_{\alpha}(d j, d k)=-\pi(d j, d k) \cdot H(j)+\bar{B}_{\alpha}(d j, d k) .
$$

This allows us to split $F(d j, d k)$ into

$$
F(d j, d k)=\vec{F}(d j, d k)-\pi(d j, d k) \cdot D V(j)
$$

where $V(j)$ is the volume of $j(M)$. Both of these equations hold for all $d j, d k$ and $\mathrm{dh}$. Notivated by the last equation we call $\pi(\mathrm{dj}, \mathrm{dk})$ the volume active pressure. $\pi(d j, d k) \cdot D V(j)$ is the work needed to change the volume by $D V(j)$. Clearly this type of pressure also exists in the realm of section 3 and is clearly. not identical (in general) with $\tau_{\text {int }}(\mathrm{dj}, \mathrm{dk})$. 


\section{References :}

[Ba] A. Bastiani : Applications differentiables et varietes differentiables de dimension infinie, J. Anal. Math., $13,1-114,1964$

[Bi,1] E. Binz : Two natural metrics and their covariant derivatives on a manifold of embeddings, Wh. Math., 89, 275-288, 1980

[Bi,2] E. Binz: The description of $\mathbb{R}^{n}$-valued one forms relative to an embedding, Mannheimer Manuskripte No. 74 , Universität Vannheim, 1987

[Bi,3] E. Binz : One forms on $E\left(M, \mathbb{R}^{n}\right)$ with integral representation, Vannheimer Manuskripte No. 79, Lniversität Vannheim, 1988

$[\mathrm{Bi}, 4] \quad$ E. Binz : On the notion of the stress tensor associated with $\mathbb{R}^{\mathrm{n}}$ invariant constitutive laws admitting integral representations, ( to appear in Reports on Mathematical Physics), 1988

[Bi,5] E. Binz: Viscosity and volume active pressure, Mannheimer Manuskripte No. 82, Universität Mannheim, 1988

[Bi,6] E. Binz : Natural Hamiltonian systems on spaces of embeddings, Proceedings of the $X V$ international conference on differential geometric methods in theoretical physics, ed. H. D. Doebner, J. D. Hennig, World Scientific, Singapore, New Jersey, Hong Kong, 1987

[Bi,Fi] E. Binz, H. R. Fischer : The manifold of embeddings of a closed manifold, Lecture Notes Phys, 139, Springer-Verlag, Brrin, 1981

[Fr, hr] Frölicher, kriegl : Linear spaces and differentiation theory, John wiley, Chichester England, 1988

$[\mathrm{Gr}, \mathrm{H}, \mathrm{V}]$ W. Greub, S. Halperin, J. Vanstone : Connections, Curvature and Cohomology, I, II, Acad. Press, New Iork, 1972-73

[Gu] J. Gutknecht : Die $C_{\Gamma}^{\infty}$ - Struktur auf der Diffeomorphismengruppe einer kompakten Mannigfaltigkeit, Diss. ETH 5379, Zürich, 1977

[Go,Gui] M. Golubitshi, V. Guillemin : Stable mappings and their singularities, Graduate Texts of Mathematics, Springer GTM 14 , Beriin, 1973

[He] E. Hellinger : Die allgemeinen Ansätze der Mechanik der Kontinua, Enzykl. Math. Wiss. 4/4, 1914 
[Hi] M. W. Hirsch : Differential Topology, Springer GTM, Berlin, 1976

[kl] W. Klingenberg : Riemannian Geometry, De Gruyter Studies in Math., Berlin, 1982

[Le,Fi] W. J. Leitman, G. M. C. Fisher: The linear theory of viscoelasticity, Handbuch der Physik, VI a/3, Editors C. Truesdell, S. Flügge, Springer-Verlag, Berlin, 1965

[Mi] P. Michor : Manifolds of differentiable mappings, Shiva Math. Series, Shiva Publ., Kent, UK, 1980

[Sch] Th. Schnyder-Peter : Zur Struktur der Immersionen einer Vannigfaltigkeit in einem euklidischen Raum, Universität Zürich, 1983

[Tr,No] C. Truesdell, W. Noll : The non-linear field theories of mechanics, Handbuch der Physik, III/3, Editors C. Truesdell, S. Flügge, Springer-Verlag, Berlin, 1973

\section{Addresses of the authors:}

Prof. Dr. E. Binz

Fakultät für Mathematik und Informatik

Universität Mannheim

Seminargebäude A 5

6800 Mannheim

Prof. Dr. D. Socolescu

Fachtereich Nathematik

Lniversität haiserslautern

Erwin-Schrödinger-Straße

6750 kaiserslautern 
Index :

interne Seitenzahl

$\begin{array}{lc}\text { continiuing mechanics } & 2 \\ \text { continuity equation } & 14 \\ \text { constitutive law } & 16 \\ \text { diffeomorphism group } & 11 \\ \text { embeddings } & 10 \\ \text { Euler's equation } & 25 \\ \text { force density } & 16 \\ \quad-\text { exterior } & 52 \\ \quad \text { - interior } & 52 \\ \text { gloval theory } & 2 \\ \text { Hodge decomposition } & 19 \\ \text { integrable part } & 29 \\ \text { integral representation } & 16 \\ \text { invariance under } \mathbb{R}^{\mathrm{n}} & 26 \\ L^{2} \text {-orthogonal of mean cursature } & 58 \\ \text { mean curvature } & 13 \\ \text { Navier-Stokes type of equation } & 56 \\ \text { principal bundle of embeddings } & 11 \\ \text { stress form } & 40 \\ \text { stress terisor } & 16 \\ \text { structural viscosity } & 50 \\ \text { volume active pressure } & 58\end{array}$

